\title{
UBAT of UFFO/Lomonosov: The X-Ray Space Telescope to Observe Early Photons from Gamma-Ray Bursts
}

\author{
S. Jeong ${ }^{1,2,3}$ • M.I. Panasyuk ${ }^{4,5} \cdot$ V. Reglero ${ }^{6}$ P. Connell ${ }^{6}$ M.B. Kim ${ }^{1}$. J. Lee $^{1}$ • \\ J.M. Rodrigo ${ }^{6}$ - J. Ripa ${ }^{7}$ - C. Eyles ${ }^{6}$ - H. Lim ${ }^{1}$ - G. Gaikov ${ }^{1}$ H. Jeong ${ }^{1}$ - V. Leonov ${ }^{1}$. \\ P. Chen ${ }^{7,8}$ - A.J. Castro-Tirado ${ }^{3,9}$ - J.W. Nam ${ }^{7,8}$ - S. Svertilov, ${ }^{4,5}$ I. Yashin ${ }^{4}$. \\ G. Garipov ${ }^{4}$ M.-H.A. Huang ${ }^{10}$ • J.-J. Huang ${ }^{7}$ - J.E. Kim ${ }^{1}$ - T.-C. Liu ${ }^{7}$ V. Petrov ${ }^{4}$. \\ V. Bogomolov ${ }^{4,5}$ - C. Budtz-Jørgensen ${ }^{11}$ - S. Brandt ${ }^{11}$ - I.H. Park ${ }^{1,2}$
}

Received: 31 January 2017 / Accepted: 27 November 2017 / Published online: 7 December 2017

(C) The Author(s) 2017. This article is published with open access at Springerlink.com

\begin{abstract}
The Ultra-Fast Flash Observatory (UFFO) Burst Alert and Trigger Telescope (UBAT) has been designed and built for the localization of transient X-ray sources such as Gamma Ray Bursts (GRBs). As one of main instruments in the UFFO payload onboard the Lomonosov satellite (hereafter UFFO/Lomonosov), the UBAT's roles are to monitor the $\mathrm{X}$-ray sky, to rapidly locate and track transient sources, and to trigger the slewing of a UV/optical telescope, namely Slewing Mirror Telescope (SMT). The SMT, a pioneering
\end{abstract}

The Lomonosov Mission

Edited by Yuri Shprits, Hans Bloemen and Jim Burch

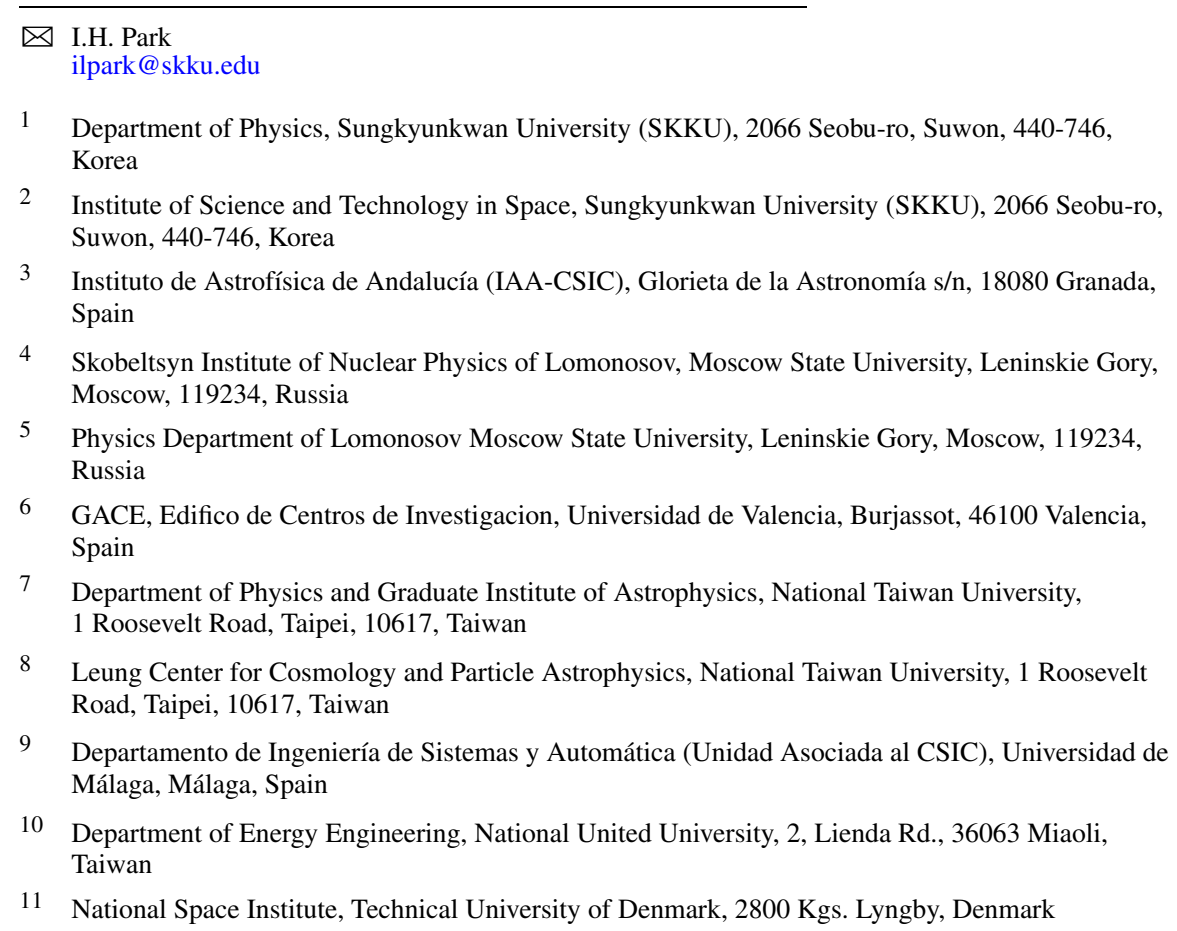


application of rapid slewing mirror technology has a line of sight parallel to the UBAT, allowing us to measure the early UV/optical GRB counterpart and study the extremely early moments of GRB evolution. To detect X-rays, the UBAT utilizes a $191.1 \mathrm{~cm}^{2}$ scintillation detector composed of Yttrium Oxyorthosilicate (YSO) crystals, Multi-Anode Photomultiplier Tubes (MAPMTs), and associated electronics. To estimate a direction vector of a GRB source in its field of view, it employs the well-known coded aperture mask technique. All functions are written for implementation on a field programmable gate array to enable fast triggering and to run the device's imaging algorithms. The UFFO/Lomonosov satellite was launched on April 28, 2016, and is now collecting GRB observation data. In this study, we describe the UBAT's design, fabrication, integration, and performance as a GRB X-ray trigger and localization telescope, both on the ground and in space.

Keywords Gamma Ray Burst · Coded mask · YSO · UFFO · Lomonosov

\section{Introduction}

Gamma Ray Bursts (GRBs) are among the most energetic and therefore intriguing phenomena in the universe. Over the last four decades, several space projects (Boella et al. 1997; Ricker et al. 2002; Gehrels et al. 2005) and various ground based telescopes (Castro-Tirado et al. 1999) have opened a new era in the exploration of increasingly earlier moments in GRB evolution with precise, rapid and deep detections of these events over a multi-wavelength band. Despite better understanding of the GRB enigma, questions of the central engine and explosion mechanism at prompt emission remain unanswered. To study the extremely early moments of GRB occurrences in terms of their UV/optical prompt emissions, the Ultra-Fast Flash Observatory (UFFO) project was proposed in 2009 (Park et al. 2009). As a pioneer mission, the UFFO/Lomonosov (Park et al. 2013, see Fig. 1) has been developed by an international consortium from Korea, Spain, Russia, Taiwan, Denmark and France.

The UFFO/Lomonosov is equipped with two main instruments: the UFFO Burst Alert and Trigger Telescope (UBAT) for X-ray detection and the Slewing Mirror Telescope (SMT) for UV/optical follow-up. The UBAT was designed and built as a wide Field of View (FOV) telescope to monitor the X-ray sky, rapidly localize transient sources such as GRBs, and issue trigger commands to the SMT. The SMT is a new UV/optical telescope of its kind of rapid reorientation of FOV, which targets sources on the axis of its focal plane within two seconds of event inception and thus measures these events' early UV/optical counterparts.

The mass and size of UBAT are $10.5 \mathrm{~kg}, 400 \mathrm{~mm}(\mathrm{w}) \times 400 \mathrm{~mm}(\mathrm{l}) \times 280 \mathrm{~mm}(\mathrm{~h})$. It is housed within the $25 \mathrm{~kg}$ of UFFO/Lomonosov package. The device contains a $191.1 \mathrm{~cm}^{2}$ scintillation detector and utilizes a coded mask correlation technique for the X-ray imaging of GRBs. The instrument's FOV covers $1 / 7$ of the sky (1.8 sr in partially coded, see Fig. 2) at an energy range of 5-200 keV. Its accuracy of angular resolution is $1.07^{\circ}$, which leads to GRB localization within an accuracy of 10 arcmin at $>7 \sigma$. The details are summarized in Table 1.

Rapid localization of GRBs within the UBAT FOV is accomplished through a fast detector-mask correlation calculation by the onboard FPGA. Following a positive identification, the UBAT passes an alert to the SMT (Jeong et al. 2013) for optical measurement of the prompt emission phase. Within several seconds of detection, the Lomonosov spacecraft will relay this alert from the UBAT to the ground via a special satellite network and, finally, to the community for follow-up observations with ground telescopes. The SMT can respond to a UBAT trigger signal within $1-2 \mathrm{~s}$ by rapidly redirecting early event photons to 


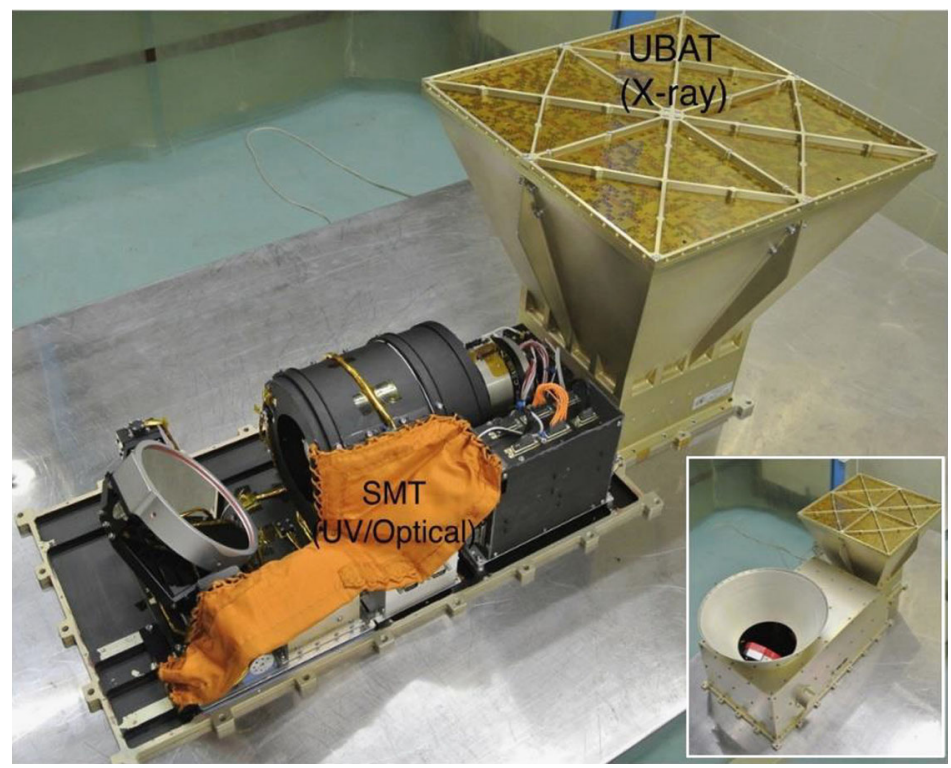

Fig. 1 Flight model of UFFO Burst and Alert Trigger telescope installed on the flight model of UFFO/Lomonosov without the SMT housing. Inset: UFFO/Lomonosov with the SMT housing. Each telescope is marked with black text

its $10 \mathrm{~cm}$ aperture Ritchey-Chrétien telescope by means of its slewing mirror system (Jeong et al. 2013; Kim et al. 2013). The Lomonosov satellite was successfully launched on April 28,2016 , has been in orbit since that time. The UBAT device was powered on several times between September 2016 and December 2016, during which times, we tested the operation of the power system and command interface. We also adjusted important UBAT parameters (thresholds for detector channels, PMT high voltages, thresholds for count rates and imaging triggers, etc.) in series of tests prior to the full operation of the UBAT.

The prompt observations of early UV/optical photons at times much closer to the preceding X-ray emissions will allow further correlation of light curves across the spectrum from optical to gamma-ray frequencies; and this, in turn, will permit further refinement of prompt emission mechanism models. The measurement of peak times within the prompt optical region will provide clues for a bulk Lorentz factor for blast waves: if the Lorentz factor is 1000, the optical telescope must respond more rapidly than $10 \mathrm{~s}$ following inception in order to observe possible peaks (see Fig. 5 in Park et al. 2013). The UFFO/Lomonosov brings us into an unexplored time domain in GRB evolution. For example, our observations are now within the onset times of the jets (which depend on the nature of the observed GRBs) as well as the transitions from prompt emission to afterglow.

In this article, we present the overall instrument details of the UBAT hardware and software, and their performance both on the ground and in space. Section 2 describes the UBAT instrument concept, design, fabrication, and assembly. The overall features of data processing and the trigger algorithm of the UBAT are reported in Sect. 3. Section 4 reports on pre-flight calibration of the UBAT instrumentation, including the uniformity of detector response to low energy X-rays and detection efficiency. The expected performance in localization of GRBs is discussed in Sect. 5. Section 6 reports briefly in-orbit operations and the conclusions provided in Sect. 7. 


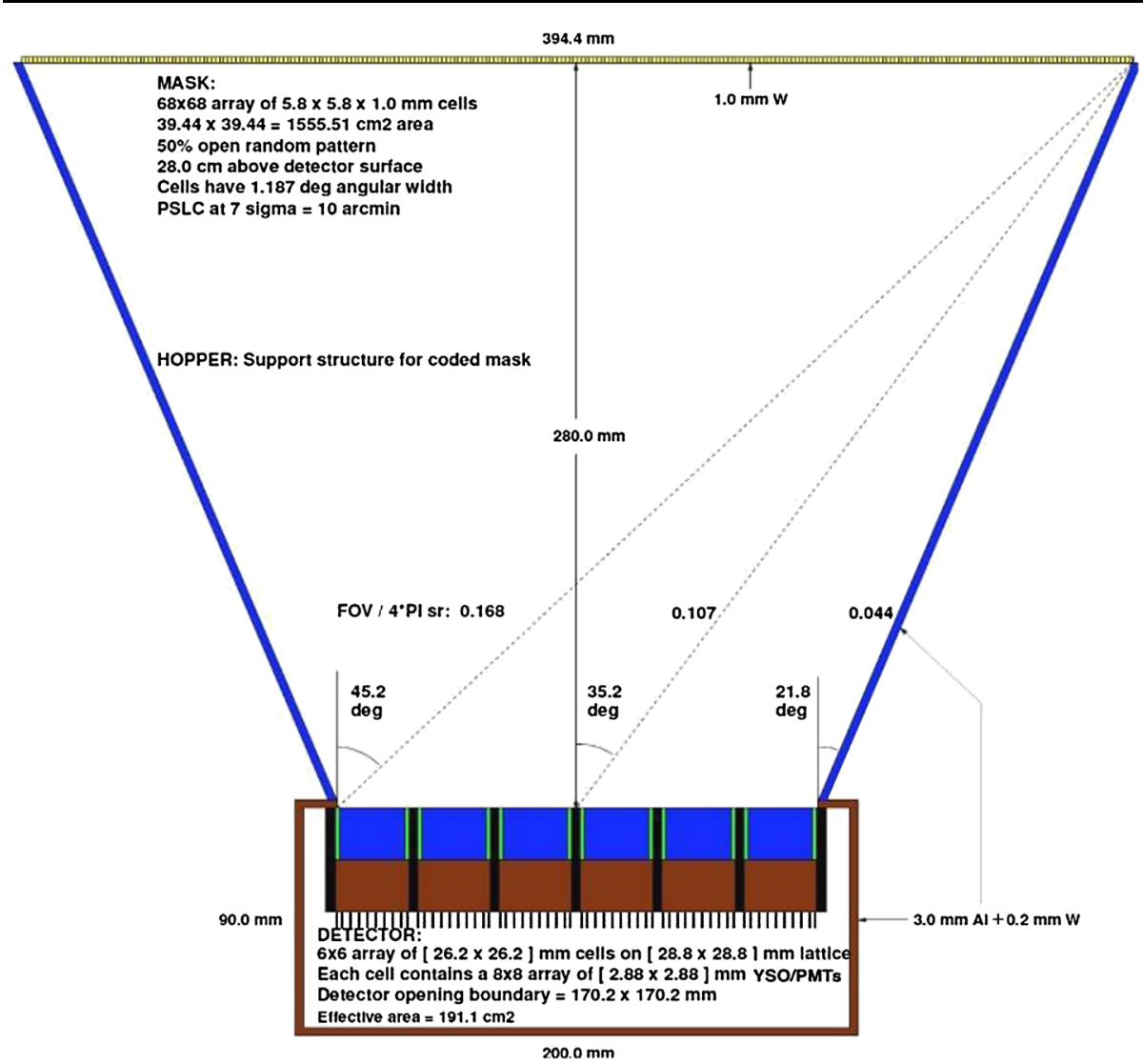

Fig. 2 Schematic drawing of the UBAT side view. The dependence of the UBAT FOV on the detector coverage is illustrated by two dotted lines extending to the lower left from the upper right corner

Table 1 UFFO/Lomonosov Burst Alert and Trigger Telescope specification

\begin{tabular}{ll}
\hline Field of view & $\sim 1.8 \mathrm{sr}$ (partially coded) \\
Detector & $6 \times 6 \mathrm{MAPMT}+$ YSO \\
Energy-band & $5-200 \mathrm{keV}$ \\
Angular resolution & $1.07^{\circ}$ \\
Pointing accuracy & $\leq 10$ arcmin accuracy for $>7 \sigma$ \\
Number of detector pixels & $48 \times 48$ \\
Pixel size & $2.88 \times 2.88 \times 3 \mathrm{~mm}^{3}$ \\
Detection area (Effective area) & $191.1 \mathrm{~cm}^{2}\left(165.5 \mathrm{~cm}^{2}\right)$ \\
Energy resolution & $2 \mathrm{keV}(\mathrm{FWHM}) \mathrm{at} 60 \mathrm{keV}$ \\
Detection efficiency & $99.4 \%$ at $30-50 \mathrm{keV}$ \\
Passive shielding composition & $0.2 \mathrm{~mm} \mathrm{~W}+3.0 \mathrm{~mm} \mathrm{Al}$ \\
Passive shielding absorption & $100 \%$ at $4-50 \mathrm{keV}$ \\
Coded mask pattern & $\mathrm{W}$ alloy of $1 \mathrm{~mm}$ thickness \\
Coded mask size & $400 \times 400 \times 1 \mathrm{~mm}^{3}$ \\
Mask to Detector & $280 \mathrm{~mm}$ \\
Weight & $10.5 \mathrm{~kg}$ \\
Volume & $400.8 \times 400.8 \times 365 \mathrm{~mm}^{3}$ \\
Average power consumption & $10 \mathrm{~W}$ \\
\hline
\end{tabular}



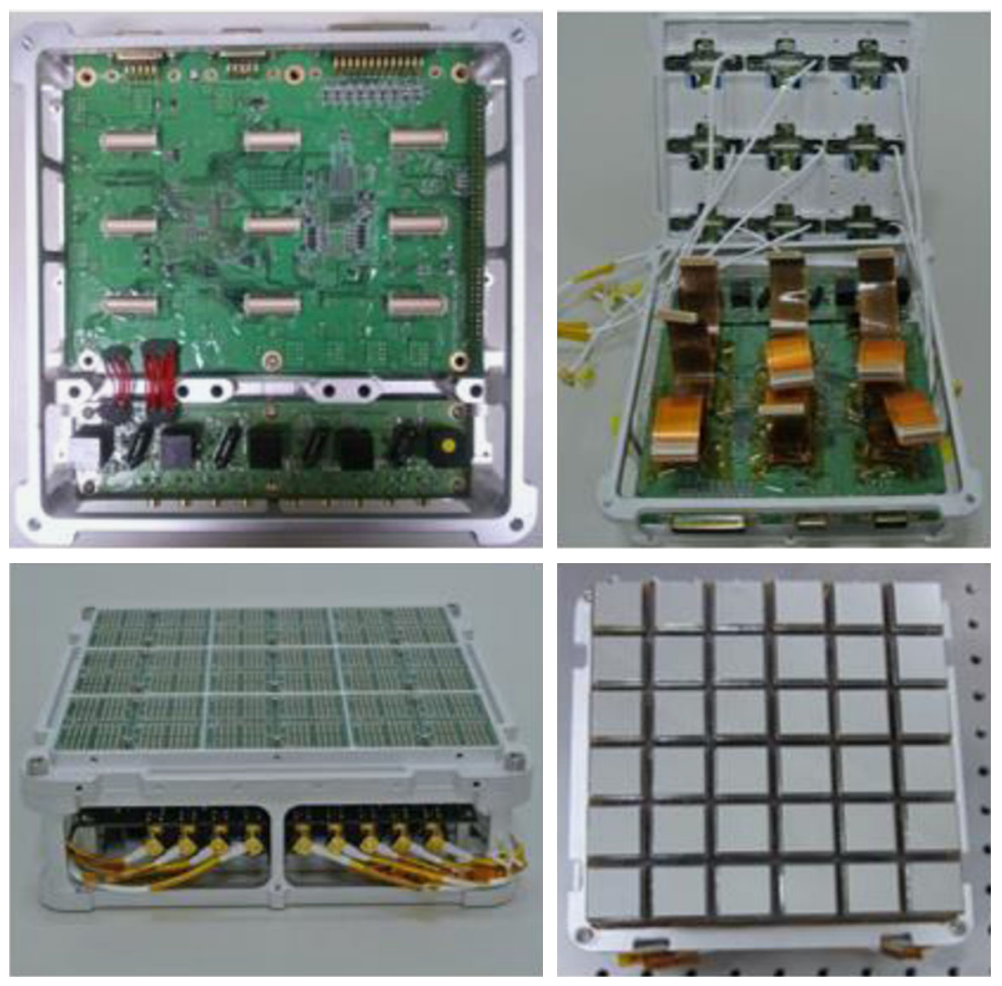

Fig. 3 UBAT detector assembly. Top left: Digital board and high voltage board. Top right: UBAT detector top and bottom parts. 9 analog boards are resident and every analog board is connected to the digital board via FPCB and to the high voltage board using high voltage cables. Bottom left: Assembly view after connection of digital board, analog boards, and high voltage board onto the UBAT detector structure. Bottom right: Integration of 36 MAPMTs and 36 YSO Scintillation crystal arrays onto the analog boards. MAPMTs are inserted directly onto analog boards using holetite

\section{Specification and Components of UBAT}

\subsection{X-ray Detector of UBAT}

The X-ray detector consists of Yttrium Oxyorthosilicate ( $\mathrm{YSO}, \mathrm{Y}_{2} \mathrm{SiO}_{5}: 0.04 \% \mathrm{Ce}$ ) scintillation crystals, Multi-Anode Photomultiplier Tubes (MAPMTs), analog, digital, and highvoltage electronics, and the assembly structure; and the total effective area of this instrument, determined by the total area of the YSO crystal layer, is $165.5 \mathrm{~cm}^{2}$. The thermal pads (Gap Pad $3000 \mathrm{SF}^{1}$ ) have been installed to fill the gaps between the heat sink and electronics devices; and the surfaces of the scintillator detectors are provided with Tedlar covers to block stray UV/optical emissions. The integrated flight model of the X-ray detector is shown in Fig. 3, and details about the individual components are described in following subsections.

\footnotetext{
${ }^{1}$ http://www.bergquistcompany.com.
} 


\subsubsection{YSO Scintillation Crystals}

The UBAT detector employs a $6 \times 6$ array of YSO crystal modules in which incident low energy X-rays are absorbed and converted to electrons by the photoelectric process in the YSO crystals. The subsequent passage of these electrons through the crystals excite the YSO atoms and produce scintillation light. Individual crystal detector modules consist of $8 \times 8$ pixel arrays that are combined to form a $48 \times 48$ pixel composite array. Individual crystal pixels are $2.68 \mathrm{~mm}(\mathrm{w}) \times 2.68 \mathrm{~mm}(\mathrm{l}) \times 3 \mathrm{~mm}(\mathrm{~h})$, but both the width and length dimensions are pitched to $2.88 \mathrm{~mm}$ to accommodate the $0.2 \mathrm{~mm}$ thick internal reflectors. These reflectors are positioned between adjacent pixels to reduce optical photon crosstalk between pixels. Individual crystal pixels are aligned and glued into place with space-qualified optical glue for attachment to the associated MAPMT array pixels. Dielectric mirror reflector films are attached to five of the six sides of each pixel cube (excluding the optical face glued to the MAPMT pixel) to reflect scintillation photons back into the crystal pixel. The $48 \times 48$ array of detector pixels effectively lie on a larger $58 \times 58$ physical lattice array when we allow for the 2 pixel size of each dead areas between the $6 \times 6$ array of YSO crystals and MAPMTs (see Fig. 2).

\subsubsection{MAPMTs}

The detection of scintillation light produced within the YSO crystals is achieved with Hamamatsu R11265-03-M64 MAPMTs. An MAPMT (23.04 mm $\times 23.04 \mathrm{~mm}$ in size) consists of an $8 \times 8$ pixel array each of size $2.88 \mathrm{~mm} \times 2.88 \mathrm{~mm}$, with no gap between pixels. Ultra-bialkali photocathodes of the MAPMT pixels absorb scintillation photons from the associated YSO crystal pixels and emit electrons via the photoelectric effect, and the multiplication of those electrons by means of a stack of metal channel dynodes results in an electric current pulse. Finally, this current is fed to the analog boards for photon counting and energy measurement.

\subsubsection{Analog Electronics}

A radiation-hardened analog board was developed to convert the photo-electron output into digital signals, to measure incident photon energies, and to take photon counts. This component utilizes a Spatial Photomultiplier Array Counting and Integrating Chip (SPACIROC) as a front-end readout Application Specific Integrated Circuit (ASIC) (Ahmada et al. 2013); this chip was specifically developed for the Japanese Experiment Module's Extreme Universe Space Observatory (JEM-EUSO) to track fluorescent light generated by Extreme Energy Cosmic Rays. The analog board has two main functions: photon counting and the measurement of energy through charge to time conversions. All data acquisition and readout are processed within a $2.5 \mu$ s gate time unit (GTU). The signals from the MAPMT anodes are fed into the ASIC preamplifiers of the analog boards and individual signals are adjusted to different gains to compensate for the non-uniform responses of the MAPMTs. If necessary, the thresholds for photon counting and energy measurement may be adjusted, even after launch. Nine analog boards in total are employed to read output signals from the $6 \times 6$ YSO and MAPMT assemblies mounted on the upper structures of the detector module as shown in Fig. 3. Each analog board contains 4 SPACIROC ASICs, each capable of processing 4 MAPMT outputs in parallel, and a circuit of 2 high voltage dividers. The analog boards are connected to digital boards and to high voltage boards, respectively, by Flexible Printed Circuit Boards (FPCBs) and high voltage coaxial cables. Each MAPMT is inserted directly 

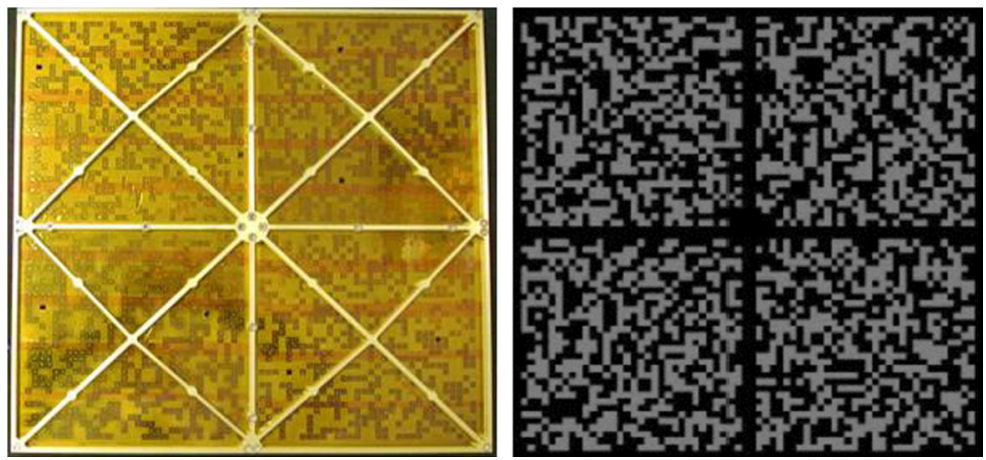

Fig. 4 Left: Optimized-random mask pattern of the tungsten coded aperture mask of the UBAT. The $1 \mathrm{~mm}$ thick coded aperture mask is reinforced with kapton tape and an aluminum spider structure. Right: The mask shadowgram pattern projecting onto the scintillator detector surface

onto the analog board through holetite socket insertion (Tyco 6-1437514-7) to minimize the detector size and to meet the UBAT height requirement, while supporting MAPMT securely under launch conditions.

\subsubsection{Digital Electronics}

Only a single UBAT digital board (presented in Fig. 3) is required for the 9 UBAT ana$\log$ boards. This component has a size of $160 \mathrm{~mm}(\mathrm{w}) \times 130 \mathrm{~mm}(\mathrm{~h})$ and plays important roles in: processing UBAT data (see Sect. 3.1); the calculation of GRB triggers (see Sect. 3.2); the regulation of driving voltage for analog boards; the control of high voltage for the MAPMTs; monitoring UBAT housekeeping temperature and voltage data; and interfacing with the UFFO/Lomonosov data acquisition system (UDAQ). To minimize the power consumption and to be suitable for space application, the digital board utilizes an Actel FPGA (A3PE3000L), which consumes $\sim 1.2-5 \mathrm{~W}$, depending on the trigger calculation stage.

\subsubsection{High Voltage Electronics}

The UBAT high voltage board employs $9 \mathrm{EMCO}^{2} \mathrm{DC}$ to $\mathrm{DC}$ high voltage converter modules to step up the voltage from the $10 \mathrm{~V}$ provided by a regulation circuit on the digital board to the 900-1000 V used in MAPMT operation. This component supplies high voltage to the MAPMTs via coaxial cables and is optimized to maintain voltages across the entire UBAT detector channels to be responded uniformly.

\subsection{Coded Aperture Mask}

The coded mask is a $1 \mathrm{~mm}$ thick tungsten (W) plate encoded by the $68 \times 68$ array of 5.76 $\times 5.76 \mathrm{~mm}^{2}$ squares of an optimized random mask (see Fig. 4). The mask is situated 280 $\mathrm{mm}$ above the UBAT scintillation detector surface and has a $395 \mathrm{~mm} \times 395 \mathrm{~mm}$ area, with each square subtending an angular width of $1.18^{\circ}$. The mask pattern has a $44.5 \%$ open fraction and is designed mathematically to cast unique shadowgrams of GRB photons onto the

\footnotetext{
${ }^{2}$ http://www.emcohighvoltage.com.
} 
Fig. 5 An exploded view of the mechanical structure of the UBAT, composed of a coded aperture mask, its reinforcing aluminum structures, and its aluminum hopper passively shielded by the $0.2 \mathrm{~mm}$ tungsten sheets lining its four sides, and UBAT detector

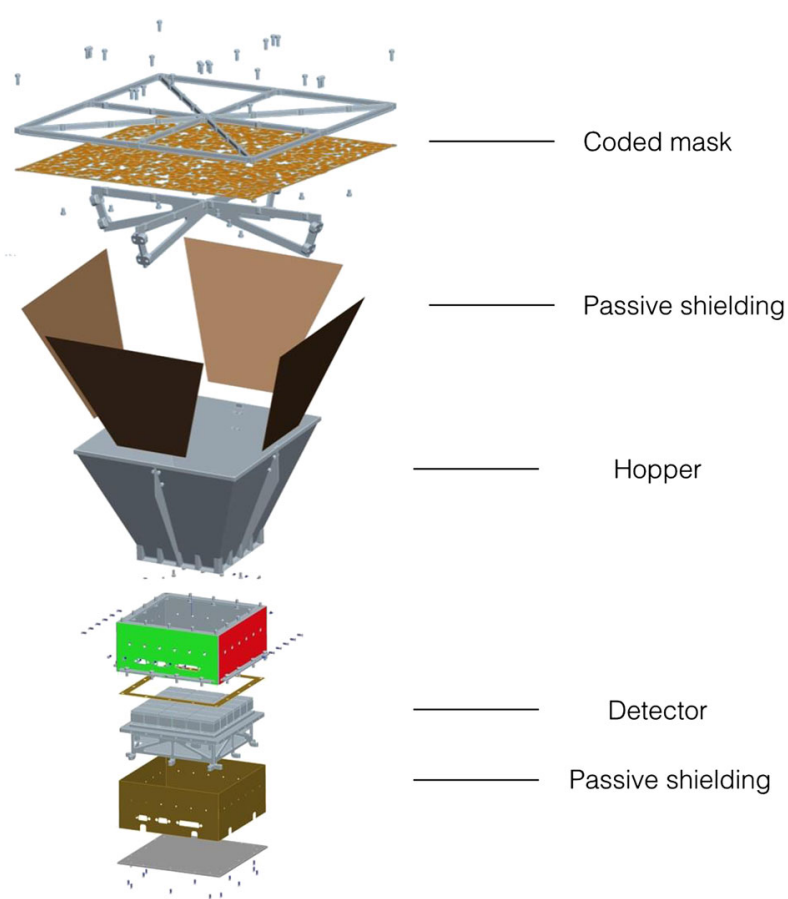

detector plane in order to determine the coordinates from which individual GRBs originate. The coded mask is reinforced by an aluminum ( $\mathrm{Al}$ ) spider structure on its lower and upper surfaces and covered with $150 \mu$ kapton $^{3}$ foil. The coded mask, its reinforced structure (see Fig. 5), and collimator have been designed and developed at the University of Valencia (Spain, Rodrigo et al. 2012) with some heritage from the INTEGRAL mission (Winkler et al. 2003).

\subsection{Collimator \& Shielding}

The UBAT collimator design is derived to accommodate the UBAT FOV through its conformity to the specified geometrical relationship of the sizes of the coded aperture mask and detector pixels and the distance intervening between them. For passive shielding against background radiation of up to $100 \mathrm{keV}$, the 4 sides of the $3.0 \mathrm{~mm}$ thick aluminum hopper are shielded on the inside by $0.2 \mathrm{~mm}$ tungsten layers (see Fig. 5). Each photon reaching the detector plane can be "back projected" through the mask, to determine the sky coordinates of its probable origin.

\section{Data Acquisition and Trigger Algorithms}

The trigger algorithm as well as data acquisition software of the UBAT are implemented into two FPGA units for the first time in space in order to minimize the trigger decision latency. These FPGAs are comprised of 3 parts: data processing unit, trigger processing unit and interface unit.

\footnotetext{
${ }^{3}$ https://www.kaptontape.com/.
} 


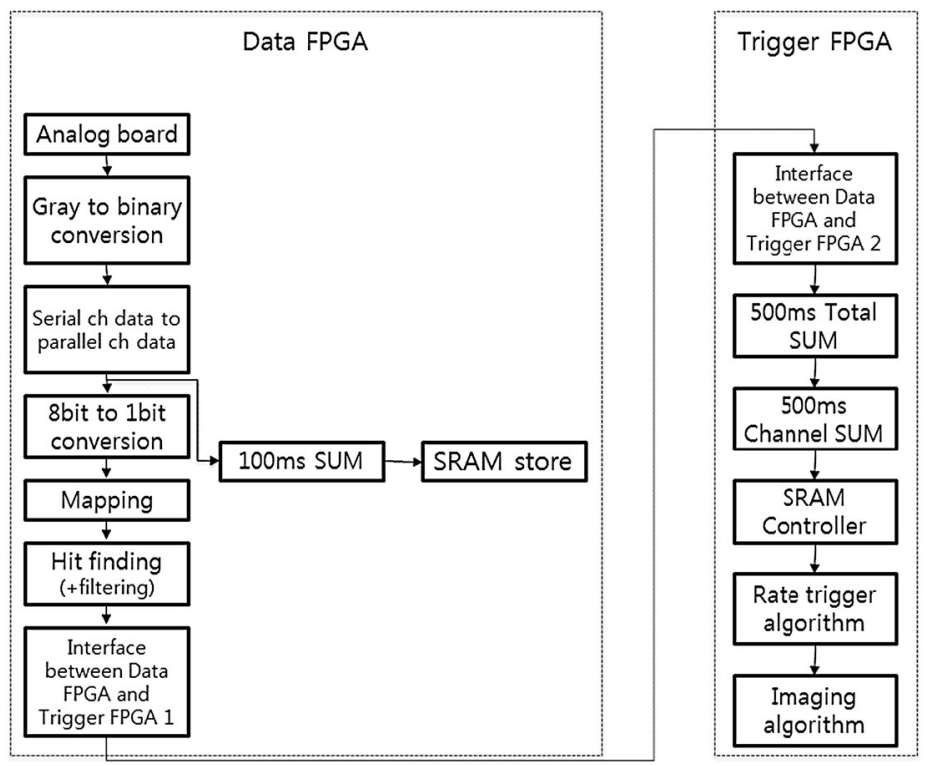

Fig. 6 Block diagram of UBAT top level data flow. The data flow is divided between two directions: raw data is stored in SRAM as are the GRB trigger calculations

Table 2 Specification of UBAT raw data depends on predefined sessions

\begin{tabular}{lcccccc}
\hline & Session 1 & Session 2 & Session 3 & Session 4 & Session 5 & Total \\
\hline Number of frames & 200 & 300 & 100 & 50 & 50 & 700 \\
Time resolution (ms) & 100 & 100 & 500 & 500 & 500 & - \\
Exposure time (s) & 20 & 30 & 10 & 25 & 25 & 110 \\
\hline
\end{tabular}

\subsection{Data Processing Unit}

A schematic diagram of the UBAT Data Processing Unit (DPU) is shown in Fig. 6. There are two flows of digital data processing. In the first flow, raw data are stored in Static Random Access Memory (SRAM) according to a predefined scheme of blocks. During space operation, the stored SRAM data will be sent to the UDAQ, and, in turn, passed to the satellite by a bus interface for transmission to the ground. The UBAT can save up to 700 frames of raw data (photon counting and energy measurement) following predefined sessions shown in Table 2. A possible storage capacity of up to $\sim 100 \mathrm{MB}$ per day is attainable through this technology, and the exposure time of individual sessions can be modified from the ground. The second data flow belongs to trigger calculation: raw data are passed to a "hit finding" or detection algorithm to remove the crosstalk that occurs when scintillation photons intrude upon neighboring MAPMT pixels, generating spurious signals. Such signals make it difficult to identify GRB coordinates via the coded mask technique; and a detailed study of mechanical crosstalk reduction is available in an excellent report by Chang et al. 2015 . However, to maintain simplicity in the design of the structural assembly of the MAPMT and YSO detectors, we decided to reduce crosstalk using a hit finding algorithm and through the adjustment of the threshold values for photon counting on the analog boards. The em- 


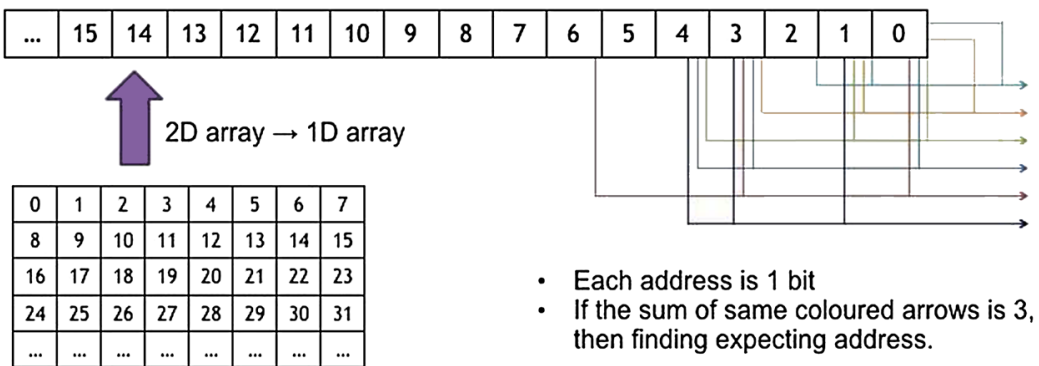
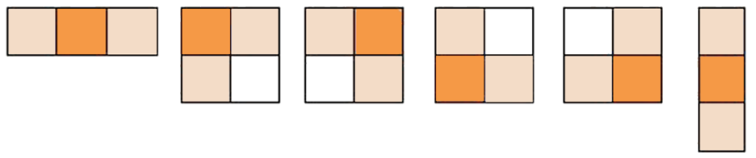

Fig. 7 Block diagram of "hit finding" algorithm for 3-hit patterns. The dark orange squares represent real hits and faint orange squares represent hits resulting from crosstalk. Serial raw data are fed into hit finding blocks, and then the finding rulers, which are represented with different colored arrows, determine the center of the hit pattern

bedded hit finding algorithm is shown schematically in Fig. 7 and uses the so-called "hit pattern recognition" technique. We have searched common crosstalk patterns after discarding patterns produced by cosmic rays, which result in simultaneous triggering across $4 \times 4$ pixels. The hit pattern recognition algorithm examines serial data to find the centers of hits immediately as they arrive. The recognition process is conducted in the Data FPGA (shown in Fig. 7) with the UBAT serving as a pipeline. The output from hit findings is sent every $500 \mathrm{~ms}$ to the Trigger Processing Unit (TPU) of the UBAT after conversion to 1 bit from 8 bits.

\subsection{Trigger Processing Unit}

A rather complicated trigger algorithm was written in VHSIC Hardware Description Language (VHDL) for implementation aboard the FPGAs, which allow for lower power consumption and faster calculation than standard CPUs. The trigger processing unit algorithm was developed and validated in the C programming language (Connell et al. 2013) prior to implementation in VHDL as the flight model system. The calculation employed by the algorithm is divided into the three following steps:

(1) Accessing a new $48 \times 48$ detector frame from the data processing unit.

(2) Saving the detector frame pixel data and the current UBAT axes matrix provided by the

Lomonosov.

(3) Executing algorithms to determine count rates and the GRB imaging trigger.

The general operation of the trigger processing unit is shown in Fig. 8 and Fig. 9. Most of the time, there will be no triggers, some false triggers, and once every several days, perhaps, triggering by an actual GRB. At that point, the task of correlation imaging begins.

From the data processing unit, $48 \times 48$ YSO-MAPMT pixel detector data are fed to the trigger processing unit continuously every $500 \mathrm{~ms}$. All channel data sums are calculated and utilized in the determination of the rate trigger, and the detector frame data is employed by the image trigger algorithm. Once the summed data fits the rate trigger criterion, the maskdetector correlation image calculation is initiated to accumulate a GRB correlation image 


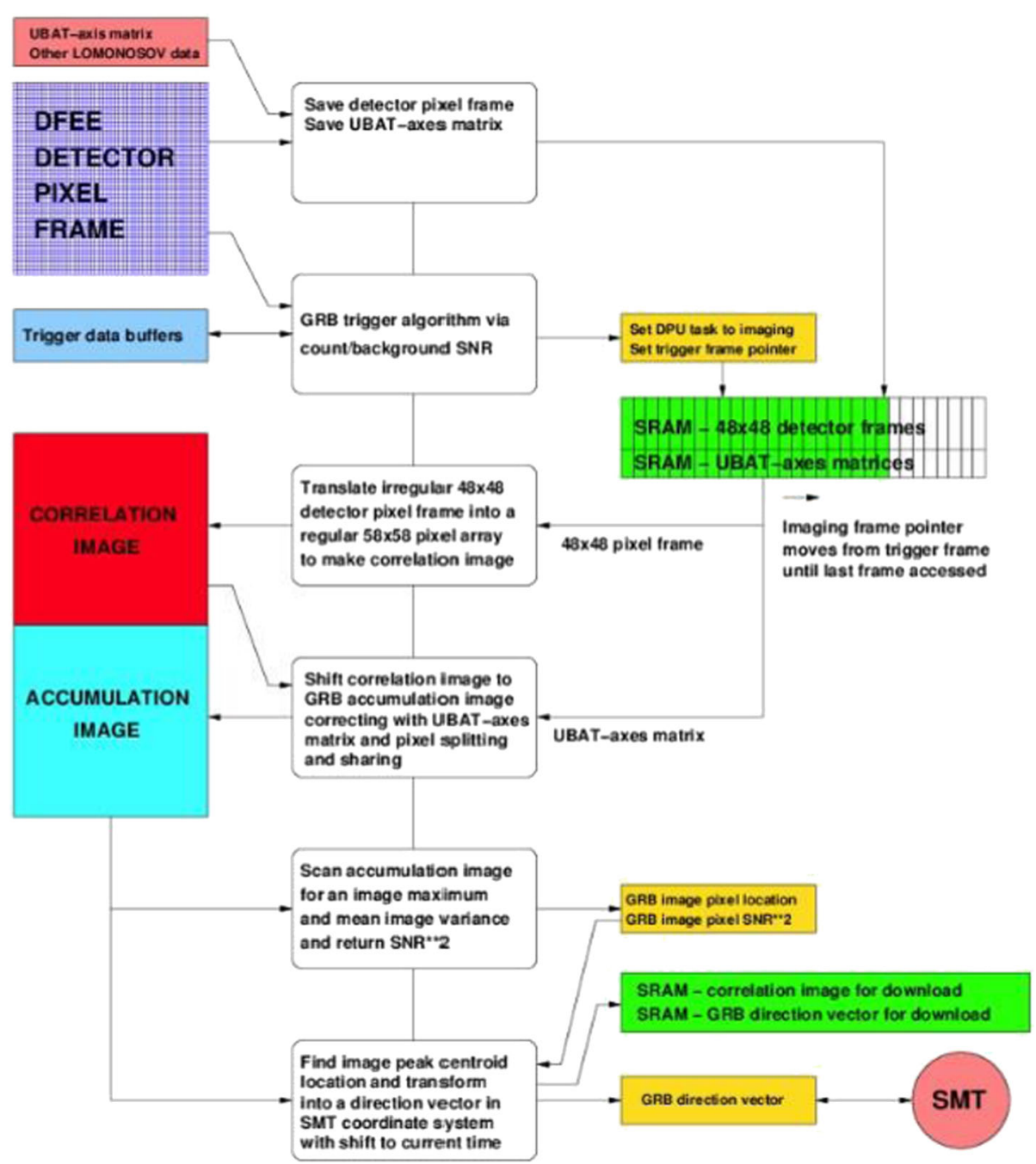

Fig. 8 General flow chart showing the multiple tasking involved in saving a $48 \times 48$ detector frame every $500 \mathrm{~ms}$, followed by the rate trigger algorithm and, finally, to the imaging algorithm, which continues until a significant GRB pixel can be found and a GRB direction vector $(X, Y, Z)$ calculated for the SMT instrument

and to search for a single significant signal to noise ratio (SNR) peak which will enable the estimation the GRB direction vector. Figure 10 shows that the Monte Carlo simulation for the required integration time to trigger for a GRB signal depends upon its incoming flux value and SNR variant. We see that a nominal GRB flux of 1 photon $/ \mathrm{cm}^{2} / \mathrm{s}$ can be triggered after a $1 \mathrm{~s}$ exposure at SNR 7.

\subsubsection{Rate Trigger}

The data is summed and fed into the "rate trigger block" every $500 \mathrm{~ms}$. The basic requirement for a rate trigger is the detection of non-statistical variations above background levels in detector count rates across a sequence of varying time width scales, 1, 2, 4, 8, 16 and $32 \mathrm{~s}$. In 

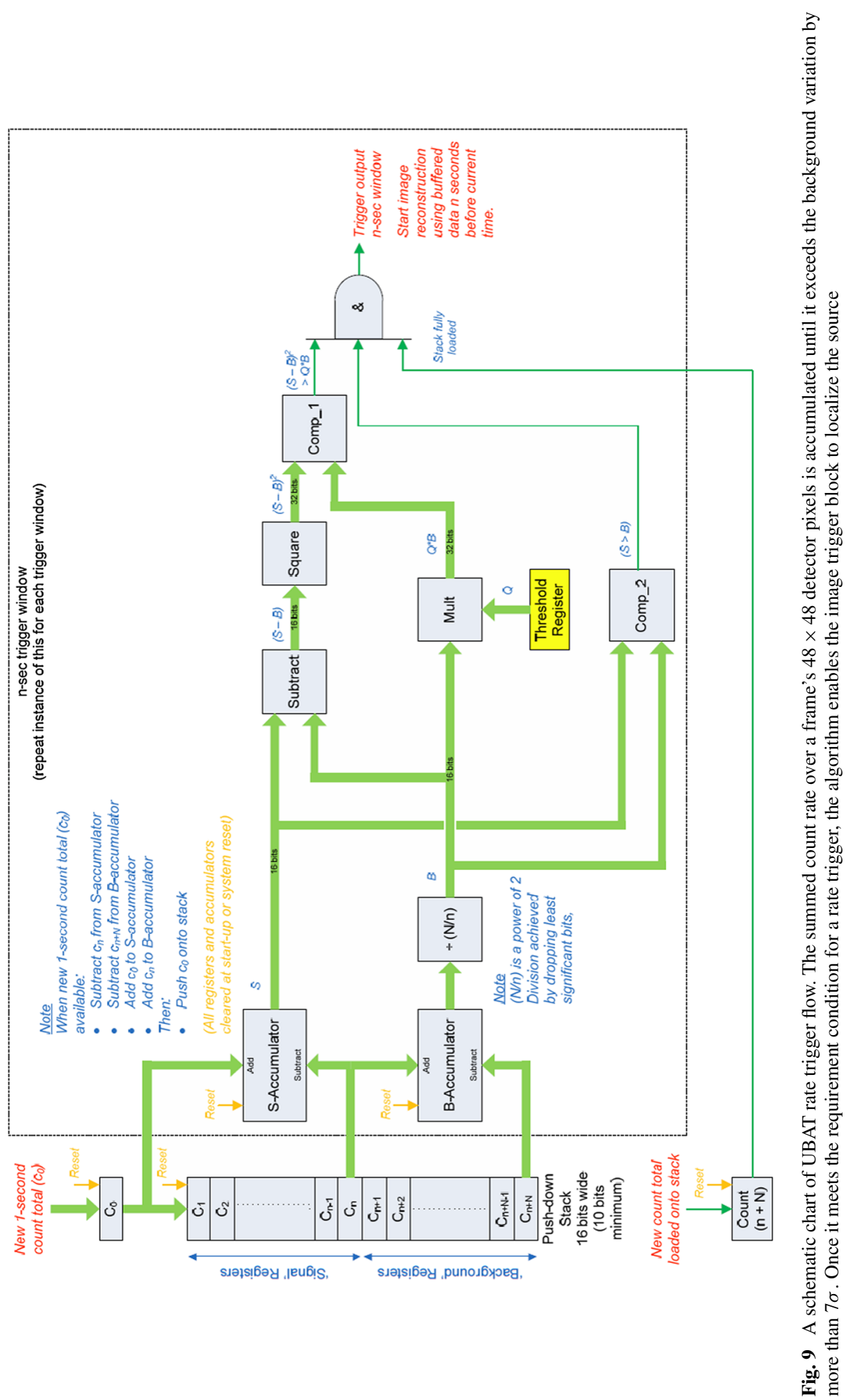


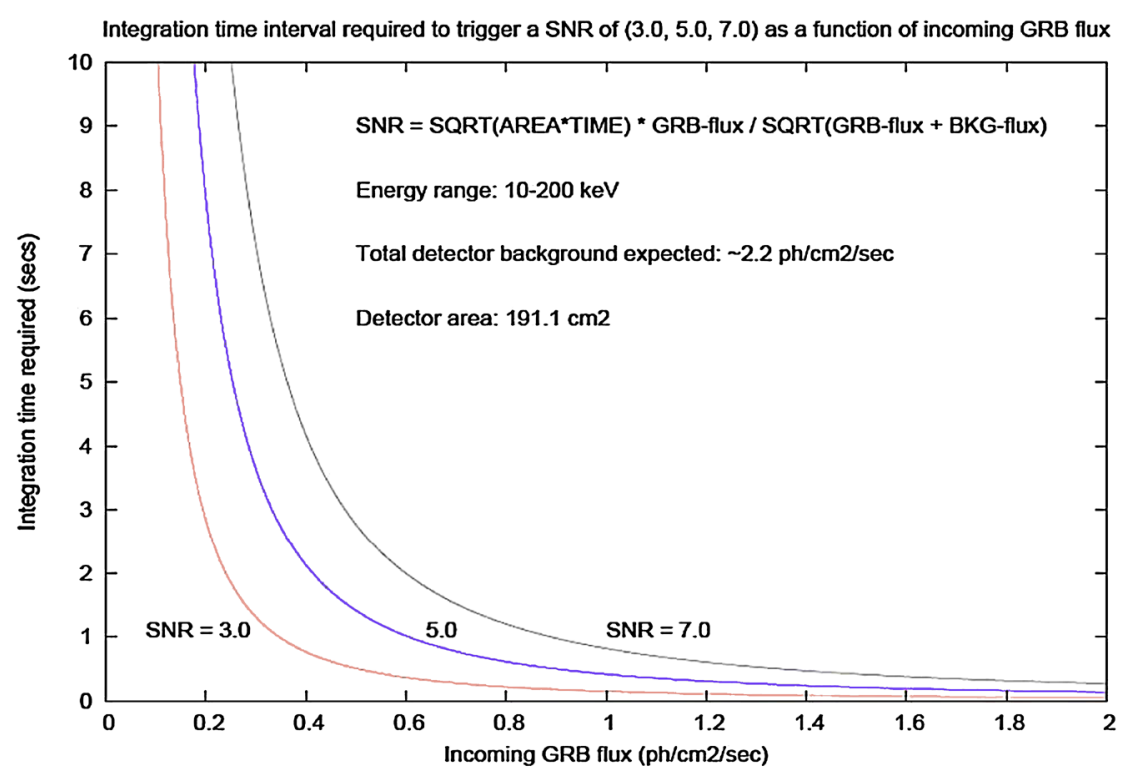

Fig. 10 The Monte Carlo simulation for the integration time required to trigger for GRB signals as a function of incoming GRB flux and SNR of $(3.0,5.0,7.0)$

order to identify a candidate GRB event, these counts are compared with background count values taken over $32 \mathrm{~s}$ and $64 \mathrm{~s}$ intervals. This is shown schematically in Fig. 9.

\subsubsection{Imaging Trigger}

To determine the GRB direction, the detector illumination pattern is imposed over the mask pattern and repositioned until the best correlation with a subset of coded mask pattern is found (Connell et al. 2013).

When a rate trigger is fired, the "imaging trigger block" executes the mask-detector correlation image reconstruction, $C[i, j]$, utilizing a $58 \times 58$ detector illumination pattern stored in a buffer. Then the imaging trigger algorithm searches a significant peak in SNR image from the variance image, $\delta^{2}[i, j]$ and correlation image $C[i, j]$ at a location of sky $[i, j]$, that is an image pixel $[i, j]$.

$$
C[i, j]=S[i, j]-O[i, j] B[i, j]
$$

where $C[i, j]$ is the correlation, $S[i, j]$ is a source image, $O[i, j]$ is a mask open fraction, and $B[i, j]$ is a background image at the image pixel $[i, j]$.

$$
\begin{aligned}
S N R^{2}[i, j] & =C^{2}[i, j] / \delta^{2}[i, j] \\
\delta^{2}[i, j] & =P[i, j] B[i, j]
\end{aligned}
$$

where $P[i, j]$ is mask coding power, defined as $P[i, j]=O[i, j](1-O[i, j]) \cong 1 / 4$. The correlation images are dependent on the location of the GRB within the UBAT FOV. The correlation imaging process for a single detector frame is described as above, but each correlation image pixel must be accumulated within a reference image by correcting for the 


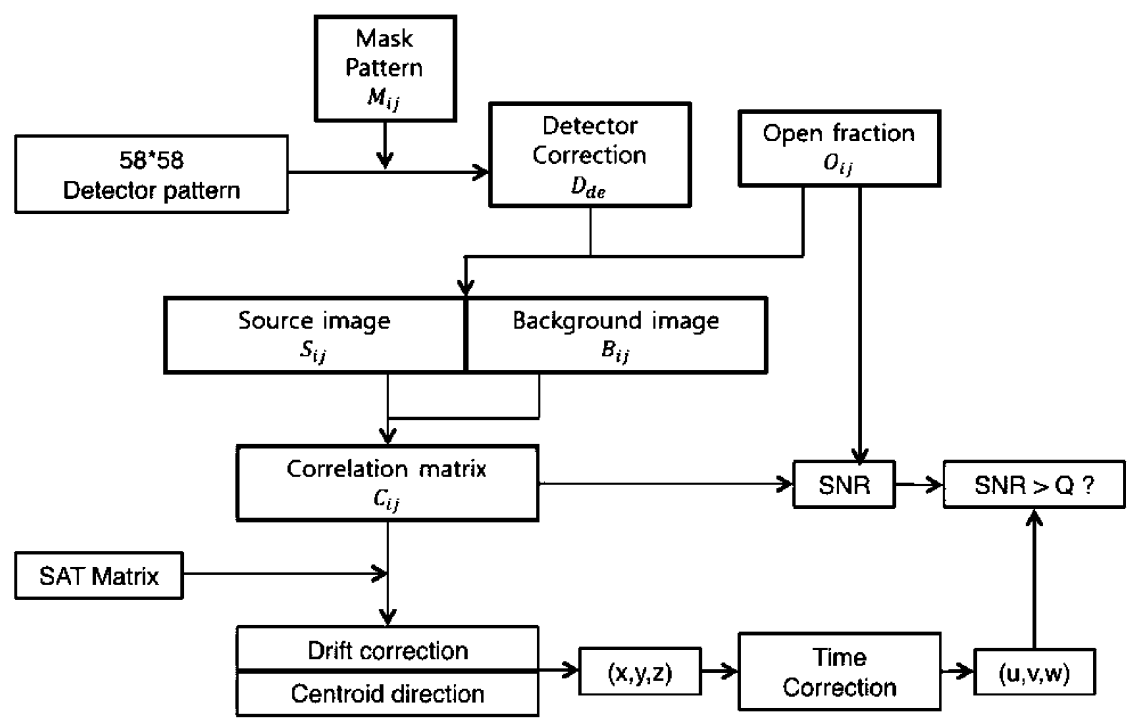

Fig. 11 A schematic chart of the UBAT imaging trigger. The $58 \times 58$ detector pattern is compared to a subset of the mask pattern to find SNR above some criterion threshold. The correlation matrix is corrected for UBAT FOV drift as the satellite rotates during an observation

UBAT FOV drift corresponding to the Lomonosov drift. The image reconstruction then continues until: (1) the SNR of the image peak reaches a predefined threshold; or (2) the SNR of the image peak reaches a maximum and then decreases by 3 steps; or (3) a time-out limit is reached. If the SNR is found to exceed $7 \sigma$ which is a rather conservative value for early stage operation but changeable after calibration of triggers, it is regarded as significant and the trigger processing unit calculates the direction vector of the source in the UBAT coordinate system and relays it to the SMT after converting the direction to that of the SMT's current frame. The UDAQ passes the trigger vector to the SMT and satellite through a bus interface in order to normalize the vector and calculate a 1-s future vector. This operation permits the optical telescope to slew to the future direction allowing for the continuous tracking of the GRB candidate and acquisition of images from the optical counterpart of the GRB. A diagram about the procedure of imaging trigger is given in Fig. 11.

\section{Pre-flight Calibration of the UBAT Detector}

\subsection{Detector Uniformity}

Response variations among the detector pixels were investigated through uniform X-ray illumination at energies of $\leq 10 \mathrm{keV}, \leq 30 \mathrm{keV}$ and $\leq 50 \mathrm{keV}$. The detector response of the UBAT is adjustable in three different ways. First, we adjusted the parameters of the ASICs' preamplifiers to change the output signal level fed into the MAPMT. Next, high voltages in the range between $942 \mathrm{~V}$ and $1044 \mathrm{~V}$ were adjusted for groups of 4 MAPMTs placed on analog boards. Finally, preamplifier output thresholds for the photon counting mode were set differently on an analog ASIC by analog ASIC basis. To generate different X-ray energies, 
Table 3 Detected count rates with a uniform illumination of the UBAT detector from different X-ray energies. The exposure time was $186 \mathrm{~s}$, frame time $10 \mathrm{~ms}$, and the area of active detector pixels $138.7 \mathrm{~cm}^{2}$

\begin{tabular}{lccl}
\hline Set-up & $\begin{array}{l}\text { Total counts } \\
(\mathrm{cnts})\end{array}$ & $\begin{array}{l}\text { Total counts-background } \\
(\mathrm{cnts})\end{array}$ & $\begin{array}{l}\text { Count rate due to the source } \\
\left(\mathrm{cnts} / \mathrm{cm}^{2} / \mathrm{s}\right)\end{array}$ \\
\hline Background & 30460 & 0 & 0 \\
$10 \mathrm{keV}$ & 320049 & 289589 & 9.72 \\
$30 \mathrm{keV}$ & 182545 & 152085 & 5.10 \\
$50 \mathrm{keV}$ & 167716 & 137256 & 4.61 \\
\hline
\end{tabular}

a Mini- $\mathrm{X}^{4} \mathrm{X}$-ray tube was placed on axis a few meters from the detector. The output of energy and flux are easily controlled by adjusting the current and voltage levels to adjust the acceleration of electrons. The Mini-X tube produces a continuum of X-rays via the Bremsstrahlung effect and yields several characteristic lines for different tube targets. The detector was exposed to X-rays of energies of $\leq 10 \mathrm{keV}, \leq 30 \mathrm{keV}$, and $\leq 50 \mathrm{keV}$ for $186 \mathrm{~s}$ (18600 frames $\times 10 \mathrm{~ms} /$ frame). The X-ray tube settings are shown below.

- X-ray energies $\leq 10 \mathrm{keV}$ : Tube voltage $10 \mathrm{kV}$, tube current $5.3 \mu \mathrm{A}$, at a source-detector distance of $4010 \mathrm{~mm}$, with no metal filter for flux attenuation.

- X-ray energies $\leq 30 \mathrm{keV}$ : Tube voltage $30 \mathrm{kV}$, tube current $5.3 \mu \mathrm{A}$, at a source-detector distance $8088 \mathrm{~mm}$, with a $\mathrm{Cu}$ filter of thickness $0.3 \mathrm{~mm}$ placed in front of the source for flux attenuation.

- X-rays energies $\leq 50 \mathrm{keV}$ : Tube voltage $50 \mathrm{kV}$, tube current $5.3 \mu \mathrm{A}$, at a source-detector distance of $8088 \mathrm{~mm}$, with a $\mathrm{Cu}$ filter of thickness $1.6 \mathrm{~mm}$ placed in front of the source for flux attenuation.

The expected X-ray spectra from the mini-X-ray tube were reproduced mathematically by solving an equation with an attenuation coefficient, ${ }^{5}$ however, without any consideration for possible secondary radiation, e.g. scattered or fluorescent X-rays. The measured detector response in terms of photon counts are presented in Fig. 12. Measured count rates are summarized in Table 3 for these X-rays. Comparing detector response from different X-ray energies with pedestal values from a non-X-ray source, we found a fraction of active detector pixels $77 \%, 94 \%$ and $95 \%$ at the X-ray energies $\leq 10 \mathrm{keV}, \leq 30 \mathrm{keV}$ and $\leq 50 \mathrm{keV}$, respectively. Pixels having values between $1 / 2$ and twice the average response values were considered active. Noisy pixels were defined as those with responses $\sim 3$ times higher than the average, and a detector pixel quality map (DPQM) was constructed by switching off noisy channels. The total fraction of noise pixels was found to be $16.2 \%$, as shown in Fig. 13 and, therefore, the effective area of the detector decreased from $165.5 \mathrm{~cm}^{2}$ to $138.7 \mathrm{~cm}^{2}$.

\subsection{Detector Efficiency}

Detector efficiency of the UBAT was studied using the onboard Block for X-ray and gammaradiation detection (BDRG) instrument. The BDRG, with its known efficiency, is one of scientific payloads onboard Lomonosov. It consists of three identical NaI(TI)/CsI(TI) (130 $\varnothing \mathrm{mm} \times 20 \mathrm{~mm}$ ) phoswich detectors (Amelushkin et al. 2013). The efficiency of the BDRG detector over $30-100 \mathrm{keV}$ is close to $100 \%$, however efficiency drops at lower energies as a

\footnotetext{
${ }^{4}$ http://www.amptek.com/products/mini-X-ray-tube/.

${ }^{5}$ http://www.nist.gov/pml/data/xraycoef/.
} 

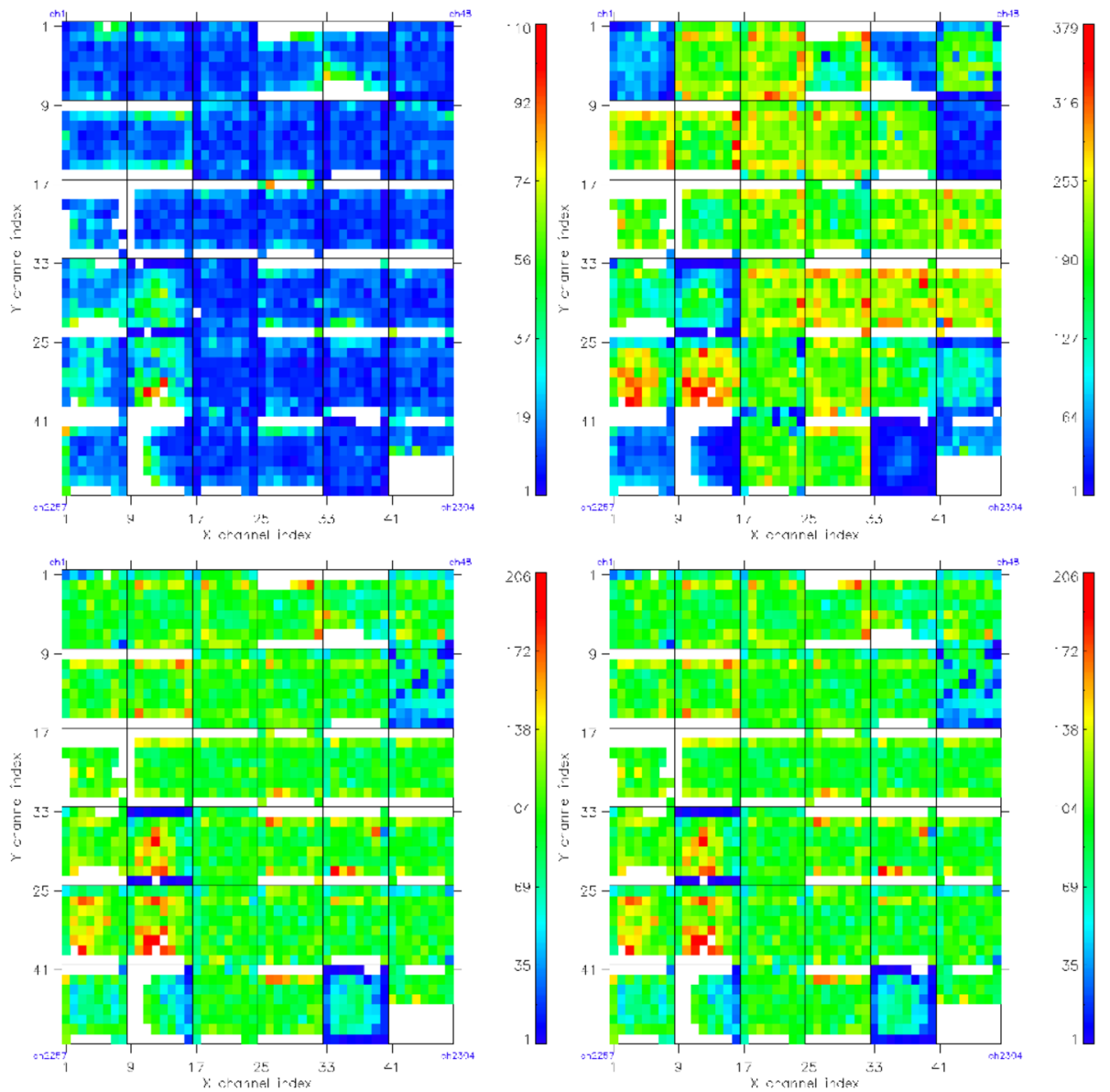

Fig. 12 Detector response of the UBAT flight model integrated over $\sim 186 \mathrm{~s}$ in a dark room. Hot, that is, noisy, channels are switched off as shown in Fig. 13. The color scale indicates the total number of counts per pixel. White indicates zero counts (resulting mainly from switched off pixels). Top left: No X-ray source, top right: X-ray energies $\leq 10 \mathrm{keV}$, bottom left: X-ray energies $\leq 30 \mathrm{keV}$, bottom right: X-ray energies $\leq 60 \mathrm{keV}$

result of the thin aluminum layer placed over the detector window to absorb the majority of $\mathrm{X}$-rays with energies below $10 \mathrm{keV}$. X-rays with energies less than $50 \mathrm{keV}$ were injected into BDRG and the UBAT mask, both at distances of $7760 \mathrm{~mm}$ from the source. The measured background rate for the BDRG from $10-100 \mathrm{keV}$ was determined to be $0.15 \mathrm{cnts} / \mathrm{cm}^{2} / \mathrm{s}$, and the count rate resulting from the X-ray was $5.18 \mathrm{cnts} / \mathrm{cm}^{2} / \mathrm{s}$. This count rate can be compared with the one measured by the UBAT detector. Table 4 shows the count rates from the various X-ray fluxes measured beneath the opened coded mask pixels. The coded mask has an open area fraction of about 0.445 : thus, the rate from source was calculated as (Total counts—background count)/(exposure time $\times$ frontal area of active detector pixels $\times 0.445)$. The count measured for the UBAT detector was $4.57 \mathrm{cnts} / \mathrm{cm}^{2} / \mathrm{s}$. Taking into consideration that flux decreases at a rate of $1 / d^{2}$, where $\mathrm{d}$ is source-detector distance, the expected count rate at the position of coded mask would be $4.96 \mathrm{cnts} / \mathrm{cm}^{2} / \mathrm{s}$. Assuming an efficiency of 
Fig. 13 UBAT detector pixel quality map for the flight model. Noise pixels at the edges of MAPMT are seen as hot channels, and blocked out for the imaging algorithm calculation. Active channels are indicated in channels of hot pixels are indicated by white. 1931 channels, or $\sim 83.8 \%$ of 2304 channels, are found to be active black and the masked-out

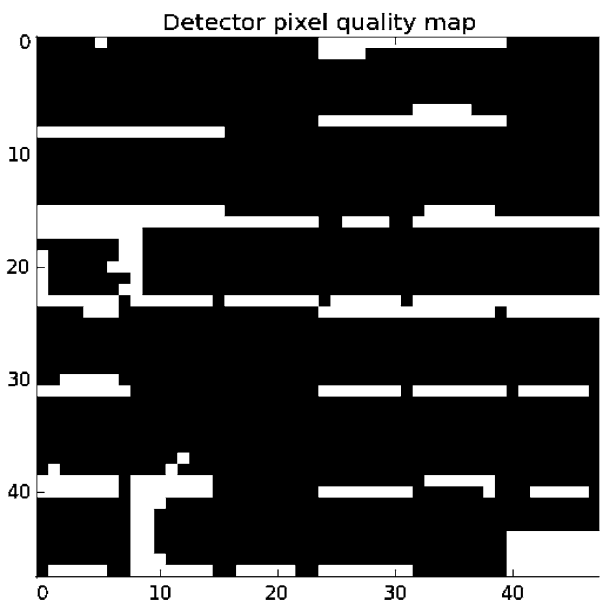

Fig. 14 Energy measurement. The $X$ axis plots the ADC count, and the $Y$ axis relative flux. One ADC count corresponds to $1.14 \mathrm{keV}$. Each set of data was taken at an exposure of $200 \mathrm{~s}$. ${ }^{241} \mathrm{Am}$ has two peaks at $18 \mathrm{keV}$ and $60 \mathrm{keV}$. The X-ray tube with appropriate parameter settings provides a spectrum with peaks at $8.6 \mathrm{keV}, 13 \mathrm{keV}$ and $26 \mathrm{keV}$

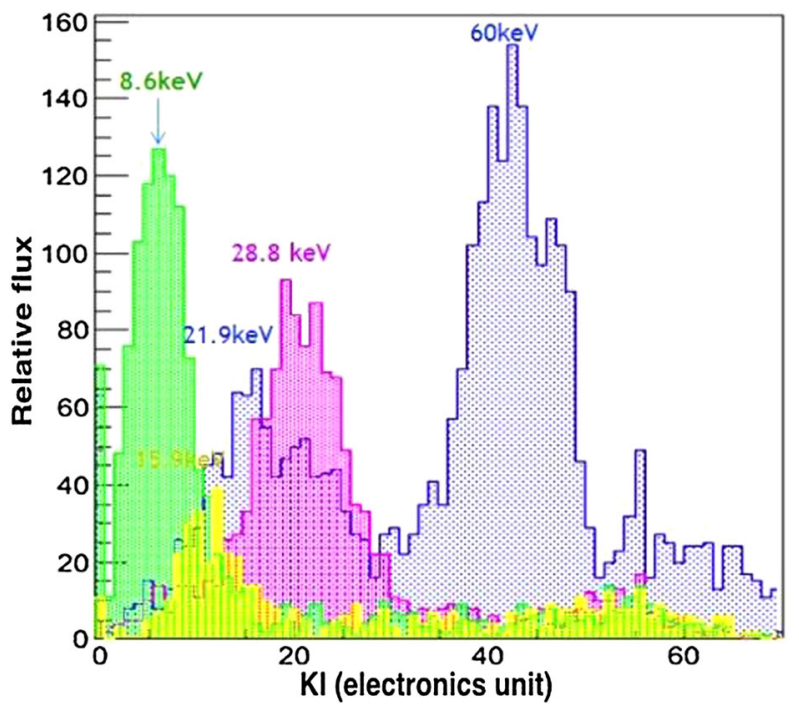

$100 \%$ for the BDRG at energies between 30 and $100 \mathrm{keV}$, the detection efficiency of the UBAT flight model is $4.96 / 5.18=95.90 \%$ over the energy range of $30-50 \mathrm{keV}$.

\subsection{Measurement of X-ray energies}

We measured energies of X-ray sources utilizing the setup described in Sect. 4.1. Because the UBAT's flight model was kept at the launch site in Russia, we conducted our preflight testing with a detector identical to the one aboard the Lomonosov. The measured spectrum is shown in Fig. 14. ${ }^{241} \mathrm{Am}$ has peaks at $18 \mathrm{keV}$ and $60 \mathrm{keV}$. The X-ray tube set to appropriate parameters provides a spectrum with peaks at $8.6 \mathrm{keV}, 13 \mathrm{keV}$, and $26 \mathrm{keV}$. Each data set was taken over a $200 \mathrm{~s}$ exposure time. The $\mathrm{X}$ axis plots the ADC count, and the $\mathrm{Y}$ axis relative flux. One ADC count corresponds to $1.14 \mathrm{keV}$. 
Table 4 The count rates measured by the UBAT detector with an effective area of $138.7 \mathrm{~cm}^{2}$ for $\leq 50 \mathrm{keV}$ $\mathrm{X}$-rays from various metal targets employed to attenuate flux. The distance between the source and detector was kept at $8090 \mathrm{~mm}$, the exposure time for each measurement was $31 \mathrm{~s}$, the frame duration is $10 \mathrm{~ms}$ with a $138.7 \mathrm{~cm}^{2}$ effective area. Background rate was $0.94 \mathrm{cnts} / \mathrm{cm}^{2} / \mathrm{s}$

\begin{tabular}{lccl}
\hline Set-up & $\begin{array}{l}\text { Total counts } \\
(\mathrm{cnts})\end{array}$ & $\begin{array}{l}\text { Total counts-background } \\
(\mathrm{cnts})\end{array}$ & $\begin{array}{l}\text { Count rate due to source } \\
\left(\mathrm{cnts} / \mathrm{cm}^{2} / \mathrm{s}\right)\end{array}$ \\
\hline Background & 4026 & 0 & 0 \\
$2.4 \mathrm{~mm} \mathrm{Cu}$ & 5023 & 997 & 0.52 \\
$2.2 \mathrm{~mm} \mathrm{Cu}$ & 5577 & 1551 & 0.81 \\
$2.0 \mathrm{~mm} \mathrm{Cu}$ & 6574 & 2548 & 1.33 \\
$1.8 \mathrm{~mm} \mathrm{Cu}$ & 8807 & 4781 & 2.50 \\
$1.6 \mathrm{~mm} \mathrm{Cu}$ & 12775 & 8749 & 4.57 \\
\hline
\end{tabular}

\section{Ground Testing of Localization Capability}

The localization capability of the UBAT with an implemented trigger software program was examined for X-ray sources of various energies and rates. A mini X-ray tube produces a variety of fluxes at X-ray energies less than $50 \mathrm{keV}$ by emulating a potential source. For an image reconstruction, a conical beam algorithm was used to include near distance effects. Five different fluxes were produced utilizing different $\mathrm{Cu}$ filter thickness values, as shown in Table 4. Figures 15-16 show imaging results. These results, the reconstructed images and source significances, were cross-checked using $\mathrm{C}$ language offline analysis software to mimic the code of trigger algorithm implemented by the FPGAs of the UBAT instrument. The reconstruction of images duplicated the process under space conditions by including the hit-finding algorithm explained in Sect. 3.1 and performing data reduction through the summation of frames and conversion of 8 bit raw data to 1 bit. The only difference between the C-based offline data processing and the onboard FPGA hit finding process, is that the former is applied every $10 \mathrm{~ms}$, and the latter, every $5 \mu \mathrm{s}$. This difference has however been negligible at the level of several photons $/ \mathrm{cm}^{2} / \mathrm{s}$ in the resultant image reconstruction, provided that the source flux remains low enough (see Table 4). The rate measured for the coded mask with the X-ray source turned off was $0.94 \mathrm{cnts} / \mathrm{cm}^{2} / \mathrm{s}$. The rates for the X-ray source turned on were $0.52,0.81,1.33,2.50$ and $4.57 \mathrm{cnts} / \mathrm{cm}^{2} / \mathrm{s}$ for which imaging with $\mathrm{SNR}=7$ was obtained in $\sim 12 \mathrm{~s}, \sim 11 \mathrm{~s}, \sim 4 \mathrm{~s}$, and $\sim 1 \mathrm{~s}$, respectively.

Figure 17 shows the result of imaging reconstruction for off-axis sources with $\leq 50 \mathrm{keV}$ $\mathrm{X}$-rays. The source was fixed and the UBAT rotated by $-30^{\circ},-15^{\circ},-5^{\circ},+5^{\circ},+15^{\circ}$, and $+30^{\circ}$ along its $Y$ axis. It is noted that off-axis angular resolution becomes better than onaxis up to a factor of about 1.4 as a result of the increased distance between the mask and the detector. Thus the localization accuracy also improves.

To test the UBAT localization accuracies, the X-ray source was placed on axis. Data drawn from the total $30 \mathrm{~s}$ of exposure were split into 15 independent sub-samples, each $2 \mathrm{~s}$ in duration. The conical beam imaging algorithm for each sub-sample were run separately. Localization accuracies depend on source significance, which is characterized by flux and accumulation time. Figure 18 shows the variance of the estimated source directions for 15 different exposures. The angular resolution (circles of $10 \mathrm{arcmin}$ and $17 \mathrm{arcmin}$ in diameter) are displayed. It is noted that the SMT FOV is $17 \times 17 \mathrm{arcmin}^{2}$. Standard deviations localized for on-axis X-ray sources along the $\mathrm{X}$ and $\mathrm{Y}$ axes are listed in Table 5. The standard deviations are calculated from 15 data samples for different SNR thresholds. The angular 

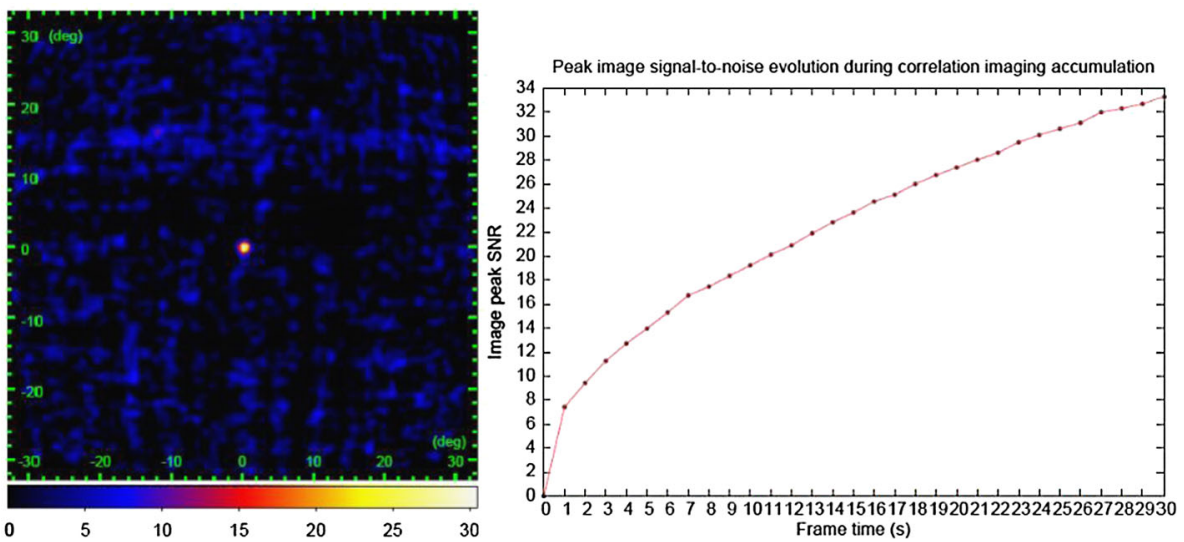

Fig. 15 Left: X-ray image reconstructed as an SNR map. The color scale refers to the SNR value. Operating at a voltage of $50 \mathrm{kV}$ and a current of $5.3 \mu \mathrm{A}$, the X-ray tube was placed on axis with the source-detector at a distance $8090 \mathrm{~mm}$, and a $1.6 \mathrm{~mm}$ thick $\mathrm{Cu}$ filter was utilized. The X-ray source is clearly visible in the middle of the figure. Right: The image peak SNR evolution during the exposure time
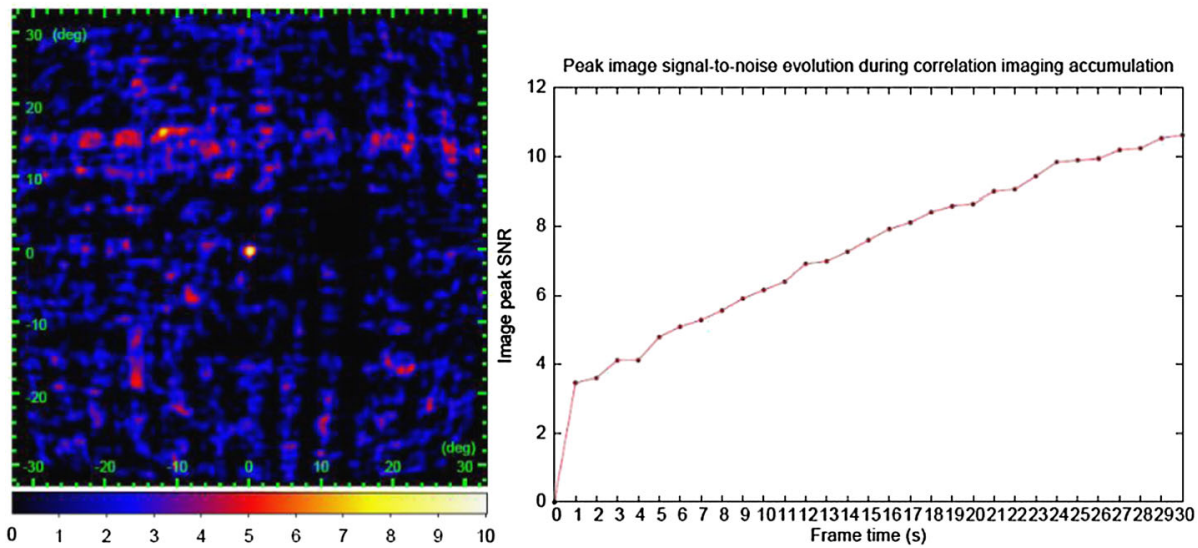

Fig. 16 Left: X-ray image reconstructed as an SNR map. The color scale refers to the SNR value. The X-ray tube was placed on axis at a voltage of $50 \mathrm{kV}$ and a current of $5.3 \mu \mathrm{A} 8090 \mathrm{~mm}$ from the source detector, and a $2.2 \mathrm{~mm}$ thick $\mathrm{Cu}$ filter was employed. The X-ray source is clearly visible in the middle of the figure. Right: The image peak SNR evolution during the exposure time

radius of the source at the distance of $8090 \mathrm{~mm}$ was $r=1.2$ arcmin, and the standard deviation of the 15 samples for the estimated directions is about $6.5 \operatorname{arcmin}$ for $\geq 7 \sigma$ detection. Thus, the localization accuracy of the UBAT is \pm 5 arcmin for $>7 \sigma$.

Laboratory confirmation was achieved for the algorithm correcting for the continual drift of detector pixels resulting from satellite movement. Correction for each pixel $C[i, j]$ occurs when the UBAT accumulates a correlation image pixel to the time of GRB trigger. To accomplish this it is necessary to convert each image pixel $[i, j]$ into a 3D pixel vector of $x, y, z$ for the UBAT coordinate system and to transform it into a set of $u, v, w$ vectors in the trigger image frame at the time of triggering, through the multiplication of a transformation matrix (the satellite matrix, which includes the UBAT of UFFO/Lomonosov position in 

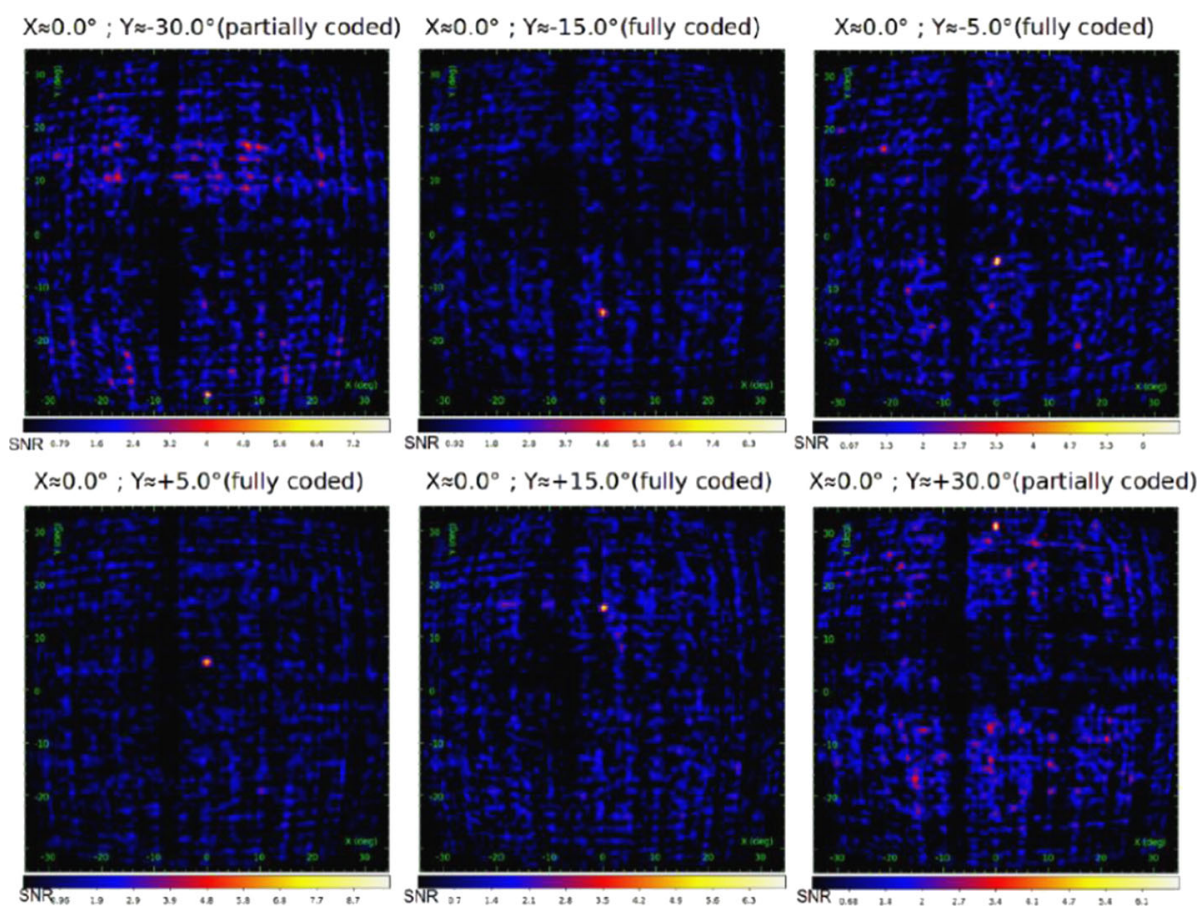

Fig. 17 Imaging reconstruction for off-axis sources with $\leq 50 \mathrm{keV}$ X-rays. The source was fixed and UBAT rotated by $-30^{\circ},-15^{\circ},-5^{\circ},+5^{\circ},+15^{\circ}$, and $+30^{\circ}$ along $Y$ axis

Table 5 The standard deviations in localization for on-axis X-ray $S N R_{\mathrm{thr}}$ sources along the $X$ and $Y$ axes are summarized. The standard deviations are calculated from 15 data samples for different SNR thresholds $\left(\mathrm{SNR}_{\mathrm{thr}}\right)$. The ranges of the SNR achieved during imaging are shown

\begin{tabular}{rlrr}
\hline$R_{\mathrm{thr}}$ & Detection SNR & $\sigma \mathrm{X}$ & $\sigma \mathrm{Y}$ \\
\hline 5 & $5.0 \sim 5.6$ & 8.2 & 10.3 \\
6 & $6.0 \sim 6.7$ & 6.9 & 9.1 \\
7 & $7.0 \sim 7.4$ & 6.5 & 6.4 \\
8 & $8.0 \sim 8.3$ & 5.5 & 6.3 \\
9 & $8.6 \sim 9.4$ & 5.1 & 6.7 \\
10 & $8.6 \sim 10.3$ & 4.3 & 5.4 \\
\hline
\end{tabular}

celestial coordinates). Next, the corresponding location of vector $u, v, w$ in the GRB trigger image must again be transformed to indicate the current time prior to relaying the trigger vectors to the SMT. For confirmation, we situated a fixed source in front of UBAT, which was also fixed. After imaging started the satellite coordinate matrix was fed into the imaging algorithm over $\sim 7-8 \mathrm{~s}$, after another satellite matrix was sent to check if UBAT finds a fixed, expected direction of $X, Y, Z$ once UBAT has determined it to be a significant source following the drift calculation. We have confirmed that the UBAT found and calculated values for $X, Y, Z$ and $u, v, w$ identical to what we expected on the basis of our mathematical calculation of vector coordinate transformations.

Long term operation of the UBAT in space may lead to the loss of detector channels. For example, there might be an issue of analog board durability for the high voltage dividers over a long operation period or because of other unforeseen conditions. Nevertheless the coded 

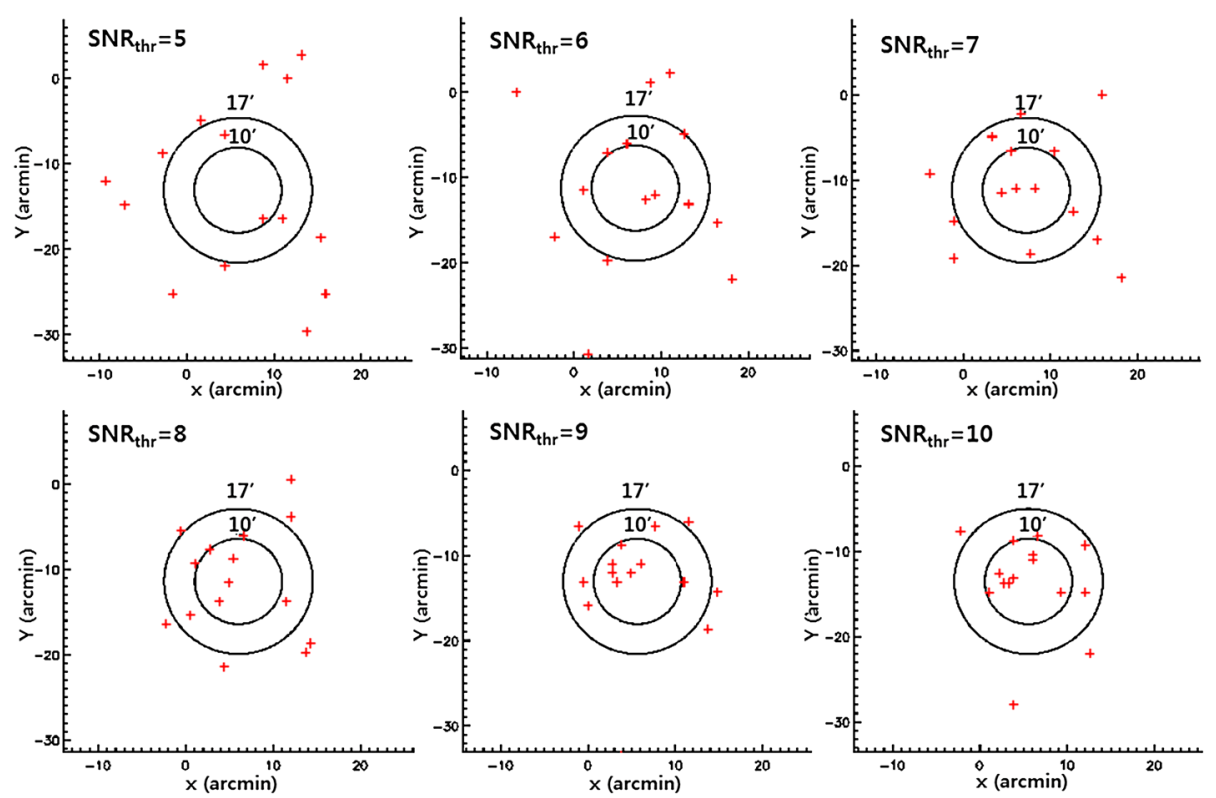

Fig. 18 Measured angular resolutions of the FM UBAT for different detection thresholds. The X-ray source was fixed and almost on-axis at distance of $8090 \mathrm{~mm}$ from the detector. The variance of the estimated source directions of 15 different exposures indicates the angular resolution. Circles of 10 arcmin and 17 arcmin in diameter are displayed. SMT FOV is $17 \times 17 \operatorname{arcmin}^{2}$

mask technique may remain relatively unaffected by any such loss of detector channels. We had proven though various lab tests such that even with the loss of $80 \%$ of the detection area, the UBAT was still able to provide meaningful triggers and localizations of an X-ray sources by permitting longer trigger latency, or longer periods of exposure prior to triggering. In this respect, a long life time is foreseen for UBAT functionality in providing triggers from space, except in cases of GRBs fading out within an order of seconds.

Figure 19 shows GRB detection sensitivity in terms of peak flux sensitivity as a function of GRB spectral peak energy $E_{\text {peak }}$ of UBAT and other missions, such as, Swift BAT (purple), SVOM (red), HETE-2 WXM (gray) and Fermi GBM (purple dot) as proposed by Band et al. 2003. The detection area of UBAT is $\sim 32$ times less than Swift/UBAT, however the sensitivity is not degraded significantly.

\section{In Orbit Operation of the UBAT}

Since the launch of the UFFO/Lomonosov in April 2016, the UBAT has been tested and calibrated 20 times by powering the instrument on. Several sets of configuration parameters were relayed from the ground for examination in space for different types of triggers including background (random) triggers and triggering by X-ray transients, such as those emitted by GRBs (physics triggers). We also tested various combinations of HV values for the MAPMTs and trigger thresholds for SNR values from 5 to 14 . We found that lowering the threshold and/or increasing HV generated false triggers fired by background reading accumulations. 


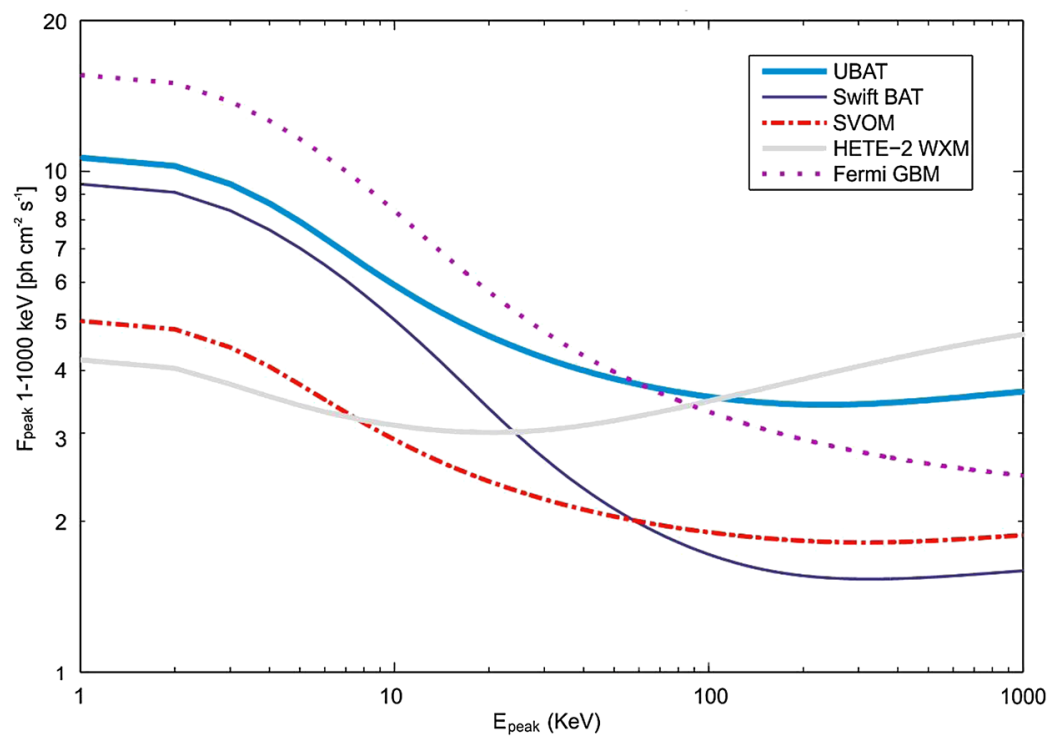

Fig. 19 The detection sensitivity of the UBAT (blue solid line) in comparison to Swift/BAT (purple solid), SVOM/ECLAIR (red), HETE-2 WXM ( gray) and Fermi/GBM (purple dot) in terms of peak flux sensitivity as a function of GRB spectral peak energy $E_{\text {peak }}$ (Band 2003; Amati et al. 2015). The GRB model with $\alpha=-1, \beta=-2$ is assumed. UBAT used $\mathrm{SNR}=7, f_{\text {det }}=0.88, \Omega=1.8$ and $f_{\text {mask }}=0.45$

The UBAT might also be exposed to non-GRB cosmic point sources and has an option to disable triggering by such known sky sources. However, this option has not been applied during any real observations, chiefly because the imaging algorithm does not take into account flaring celestial sources such as Sco X-1 on the assumption that such flares in their active states are rare events. Nevertheless they can be recognized as non-GRB sources following an analysis of UBAT data back on earth. On the other hand, during its quiescent periods, the flux of Sco X-1 is $\sim 0.1 \mathrm{cnts} / \mathrm{cm}^{2} / \mathrm{s}$ at $15 \mathrm{keV}$, which will not produce a high enough SNR for triggering within our preset timescale of $64 \mathrm{~s}$ for expected GRB duration.

With the programmed settings for random triggers, diffuse $\mathrm{X}$-ray backgrounds were measured on 12 occasions, each time for total of 700 frames, with frame exposures of $1 \mathrm{~ms}, 100$ $\mathrm{ms}$, and $500 \mathrm{~ms}$. Figure 20 shows the measurement of diffuse X-ray backgrounds. The left plot is a hit map covering 2304 detector channels, each channel summed over 700 frames. It is noted that first 500 frames have exposures of $100 \mathrm{~ms}$, while the rest have exposures of $500 \mathrm{~ms}$. Noisy channels, colored pink in the figure, are masked by programming so as not to contribute to the calculation of a trigger. The right plot shows total measured X-ray counts for the detector plotted against time. Count rates double after $50 \mathrm{~s}$ because of the doubling of the exposure time.

The X-ray backgrounds in space are extracted from the data in Fig. 20 and found to be $7 \mathrm{cnts} / \mathrm{cm}^{2} / \mathrm{s}$. The crosstalk among YSO detector pixels is known to yield three times more hits. As described in Sect. 3.1, this crosstalk is typically observed across clusters of adjacent pixels and removed from trigger calculations by a dedicated "hit finding" algorithm, implemented by the FPGAs. On the other hand, the UBAT is sensitive to low energy X-rays, down to $5 \mathrm{keV}$, and thus collects about 2.5 times more background events in comparison to the CZT detector of the Swift BAT, which is sensitive down to $15 \mathrm{keV}$. We have analyzed our data recently and found that cosmic ray interactions constitute an additional source of 

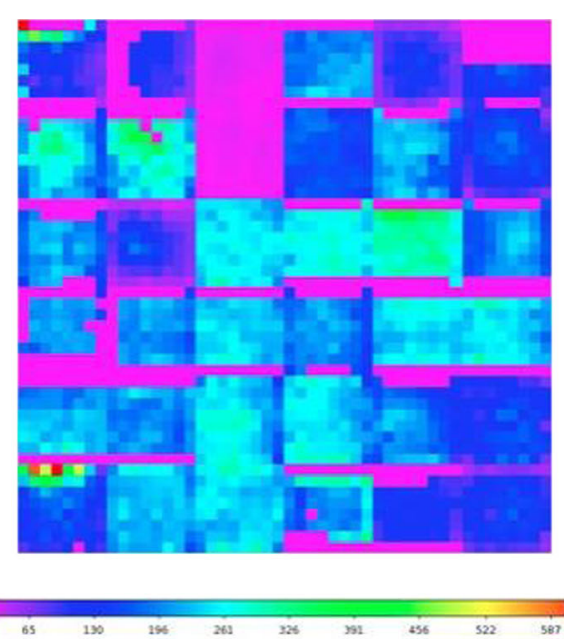

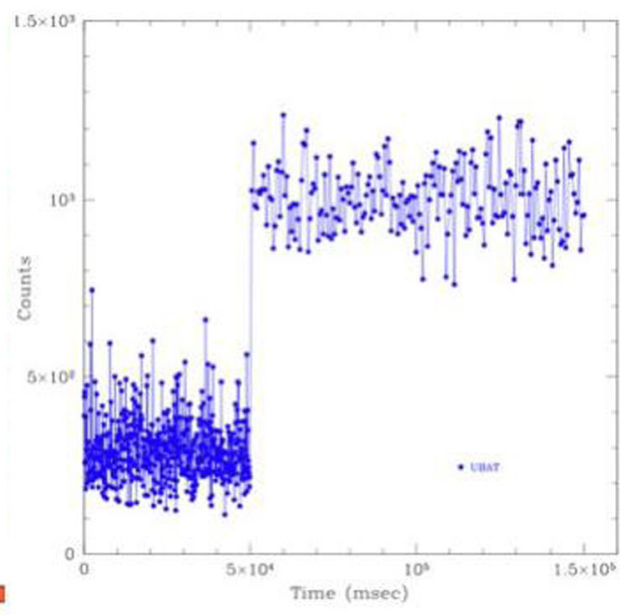

Fig. 20 Left: UBAT detector hit map for $48 \times 48$ channels summed over 700 frames. The channels colored pink were found to be noisy and, therefore, masked so that they do not contribute in trigger calculations. Right: Detected X-ray background counts plotted against time on the UBAT detector. Count rates double after $50 \mathrm{~s}$ because the frame exposure times are also doubled. The X-ray background counts in space turns out to be about $21 \mathrm{cnts} / \mathrm{cm}^{2} / \mathrm{s}$, which is about three times larger than the values we measured on the ground prior to launch

detector hits. These events typically generate hits in the form of large clusters, however we have found that they increase total hits only by a factor of 1.1. On the other hand, the difference in background counts between space and ground turns out to be $1.1 \times 2.5=2.75$, or about three times.

\section{Conclusions}

The UBAT X-ray imager utilizes a coded aperture mask, which has been designed, constructed, and put through a series of tests in space to prove its capability in detecting and imaging X-ray transient sources such as GRBs. The YSO scintillation crystal array is introduced for the first time as an imaging detector for the localization of GRBs during space observation. The detector response was made uniform over all detector channels through the adjustment of various detector operation parameters, and X-ray source tests were performed for various configurations of source and detector.

We demonstrated the triggering and imaging capabilities of the UBAT on ground, and the accuracy of localization was measured and found to be close to the design expectation. Calculations of excess in count rates and the imaging of X-ray sources together with a real time correction of satellite movement (drift correction) was successfully implemented through the onboard FPGA IC chips of the UBAT system. This is the first time ever utilization of FPGAs, which permit the much faster implementation of X-ray imaging algorithms (less than a second) and lower power consumption (less than $2 \mathrm{~W}$ ). This is a significant improvement over conventional CPU-based technology. Therefore, total time required for triggering a typical GRB is now on the order of 1-2 seconds, even for a relatively small detector like the UBAT.

The degradation of sensitivity is inevitable with a small or loss of detection area for a given observing time. It is noted that when longer times are allowed for triggering, the 
location accuracy is not seriously affected. Long accumulation times effectively compensate small detection areas, except in cases where the GRB fades out within an order of seconds. The UBAT was designed with a trigger latency of typically one second with the goal of detecting GRBs with an apparent brightness of 1 photon $/ \mathrm{cm}^{2} / \mathrm{s}$. In such cases the UBAT will definitely trigger within a couple of seconds, including less than a half second for the imaging calculation by the fast FPGAs. On the other hand, for the same source, $0.03 \mathrm{~s}$ would be sufficient for imaging in Swift BAT because its detection area is larger by a factor of 32 . However, this rapid accumulation of photons by the Swift device is subsequently followed by an imaging calculation of typically $5 \sim 6 \mathrm{~s}$. Consequently, as long as detection occurs within an order of seconds, the longer accumulation times of a relatively small detection area would not constitute a serious drawback for contemporary GRB localization technology.

The Lomonosov satellite was successfully launched into space on April 28, 2016, and it has been in the orbit since that time. The UBAT aboard the UFFO/Lomonosov went through a careful initial check-up process in orbit in preparation for operation with actual GRB triggers. During the early phase of instrument calibration, we have measured and understood the diffuse X-ray background and examined a series of trigger schemes through the adjustment of programmable instrument parameters, including thresholds, exposure time, and HV values. The details of our X-ray measurements and data analysis are now under way and are being prepared for subsequent publication.

Acknowledgements The Korean work is supported by the National Research Foundation grants funded by MSIP of Korea (Creative Research Initiatives program for RCMST, No. 2015R1A2A1A01006870, and No. 2015R1A2A1A15055344). SJ acknowledges the support of the Korea Basic Science Research Program through NRF-2015R1D1A4A01020961. MBK acknowledges support from the NRF-2015-Global Ph.D. Fellowship Program in Korea. The Russian work was partially supported by ROSCOSMOS grants and by RFFI grants No. 13-02-12175 and No.15-35-21038 and also acknowledges support from the Development Program of Lomonosov Moscow State University. AJCT acknowledges support from the Spanish MINECO Projects AYA 2009-14000-C03-01/ESP and AYA 201571718R (including EU/FEDER funds). The Taiwan authors thank Taiwan's National Science Council Vanguard Program (100-2119-M-002-025) as well as the Ministry of Science and Technology (MOST) for its funding (104-2811-M-002-160). We also acknowledge the support of the National Space Organization (NSPO) of Taiwan.

Open Access This article is distributed under the terms of the Creative Commons Attribution 4.0 International License (http://creativecommons.org/licenses/by/4.0/), which permits unrestricted use, distribution, and reproduction in any medium, provided you give appropriate credit to the original author(s) and the source, provide a link to the Creative Commons license, and indicate if changes were made.

\section{References}

S. Ahmada, P. Barrillona, S. Blin-Bondila, S. Dagoret-Campagnea, C. de La Taillea, F. Dulucqa, G. MartinChassarda, Y. Kawasakib, H. Miyamotob, H. Ikedac, T. Iguchid, F. Kajinod, SPACIROC2: a front-end readout ASIC for the JEM-EUSO observatory. J. Instrum. 8, 01006 (2013)

L. Amati, G. Stratta, J.-L. Atteia, M. De Pasquale, E. Del Monte, B. Gendre, D. Götz, C. Guidorzi, L. Izzo, C. Kouveliotou, J. Osborne, A.V. Penacchioni, P. Romano, T. Sakamoto, R. Salvaterra, S. Schanne, J.J.M. in't Zand, L.A. Antonelli, J. Braga, S. Brandt, N. Bucciantini, A. Castro-Tirado, V. D’Elia, M. Feroci, F. Fuschino, D. Guetta, F. Longo, M. Lyutikov, T. Maccarone, V. Mangano, M. Marisaldi, S. Mereghetti, P. O’Brien, E.M. Rossi, F. Ryde, P. Soffitta, E. Troja, R.A.M.J. Wijers, B. Zhang, Probing the emission physics and weak/soft population of Gamma-Ray Bursts with LOFT (2015). arXiv:1501.02772

A.M. Amelushkin, V.V. Bogomolov, V.I. Galkin, B.V. Goncharov, E.S. Gorbovskoy, V.G. Kornilov, V.M. Lipunov, M.I. Panasyuk, V.L. Petrov, G.F. Smoot, S.I. Svertilov, N.N. Vedenkin, I.V. Yashin, BDRG and SHOK instruments for study of GRB prompt emission in Michaylo Lomonosov space mission. EAS Publ. Ser. 61, 553-559 (2013)

G. Boella, R.C. Butler, G.C. Perola, L. Piro, L. Scarsi, J.A.M. Bleeker, BeppoSAX, the wide band mission for X-ray astronomy. Astron. Astrophys. 122, 299B (1997) 
A.J. Castro-Tirado, J. Soldán, M. Bernas, P. Páta, T. Rezek, R. Hudec, T.J. Mateo Sanguino, B. de La Morena, J.A. Berná, J. Rodríguez, A. Penã, J. Gorosabel, J.M. MásHesse, A. Giménez, The burst observer and optical transient exploring system (BOOTES). Astron. Astrophys. 138, 583 (1999)

Y.-Y. Chang, C.R. Chen, P. Chen, J.-J. Huang, M.A. Huang, T.-C. Liu, J.W. Nam, M.-Z. Wang (UFFO collaboration), Inverted-conical light guide for crosstalk reduction in tightly-packed scintillator matrix and MAPMT assembly. Nucl. Instrum. Methods Phys. Res. 771, 55-65 (2015).

P.H. Connell, V. Reglero (UFFO collaboration), The Ultra Fast Flash Observatory pathfinder-UFFO-p GRB imaging and location with its coded mask X-ray imager UBAT. EAS Publ. Ser. 61, 517-523 (2013)

N. Gehrels, G. Chincarini, P. Giommi, K.O. Mason, J.A. Nousek, A.A. Wells, N.E. White, S.D. Barthelmy, D.N. Burrows, L.R. Cominsky, The Swift Gamma-Ray Burst mission. Astrophys. J. 621, 558 (2005)

S. Jeong, J.W. Nam, K.-B. Ahn (UFFO collaboration), Slewing Mirror Telescope optics for the early observation of UV/optical photons from Gamma-Ray Bursts. Opt. Express 21, 2263 (2013)

J.E. Kim, H. Lim, J.W. Nam (UFFO collaboration), Readout of the UFFO Slewing Mirror Telescope to detect UV/optical photons from Gamma-Ray Bursts. J. Instrum. 8, P07012 (2013)

I.H. Park (UFFO collaboration), Ultra-fast flash observatory for the observation of early photons from Gamma-Ray Burst. New J. Phys. 15, 023031 (2013)

I.H. Park, B. Grossan, H. Lim, J.W. Nam, P. Chen, B.A. Khrenov, Y.-K. Kim, C.-H. Lee, J. Lee, E.V. Linder, M. Panasyuk, J.H. Park, G.F. Smoot, Z.L. Uhm, The UFFO (Ultra- Fast Flash Observatory) pathfinder (2009). arXiv:0912.0773

G.R. Ricker, R.K. Vanderspek, H.S. Team, GRBs from the HETE mission: recent results and future prospects from the HETE mission: recent results and future prospects. Bull. Am. Astron. Soc. 34, 1220 (2002)

J.M. Rodrigo, J.M. Maci’an, J.T. Biosca, M. Reina, L. Sabau-Graziati, V. Reglero, UFFO Burst Alert \& Trigger Telescope (UBAT): a new instrument for GRBs detection. Mem. Soc. Astron. Ital. 83, 370 (2012)

C. Winkler, T.J.-L. Courvoisier, G.D. Cocco, N. Gehrels, A. Gimènez, S. Grebenev, W. Hermsen, J.M. MasHesse, F. Lebrun, N. Lund, G.C. Palumbo, J. Paul, J.-P. Roques, J.H. Schnopper, V. Schönfelder, R. Sunyaev, B. Teegarden, P. Ubertini, G. Vedrenne, A.J. Dean, The INTEGRAL mission. Astron. Astrophys. 411, 1-6 (2003)

D.L. Band, Comparison of the Gamma-Ray Burst sensitivity of different detectors. Astrophys. J. 588, 945951 (2003) 